\title{
Interday Forecasting and Intraday Updating of Call Center Arrivals
}

\author{
Haipeng Shen • Jianhua Z. Huang \\ Department of Statistics and Operations Research, University of North Carolina at Chapel \\ Hill, Chapel Hill, NC 27599, USA \\ Department of Statistics, Texas A\&M University, College Station, TX 77843, USA \\ haipeng@email.unc.edu•jianhua@stat.tamu.edu
}

\begin{abstract}
Accurate forecasting of call arrivals is critical for staffing and scheduling of a telephone call center. We develop methods for interday and dynamic intraday forecasting of incoming call volumes. Our approach is to treat the intraday call volume profiles as a highdimensional vector time series. We propose to first reduce the dimensionality by singular value decomposition of the matrix of historical intraday profiles and then apply time series and regression techniques. Both interday (or day-to-day) dynamics and intraday (or withinday) patterns of call arrivals are taken into account by our approach. Distributional forecasts are also developed. The proposed methods are data-driven, and appear to be robust against model assumptions in our simulation studies. They are shown to be very competitive in outof-sample forecast comparisons using two real data sets. Our methods are computationally fast and therefore it is feasible to use them for real-time dynamic forecasting.
\end{abstract}

Keywords dimension reduction, dynamic forecast updating, principal component analysis, penalized least squares, singular value decomposition, vector time series.

\section{Introduction}

Call centers have become a primary contact point between service providers and their customers in modern business. For example, it is estimated that in 2002 more than $70 \%$ of all customer-business interactions are handled in call centers, and the call center industry in US employs more than 3.5 million people, or 2.6\% of its workforce (Brown et al. 2005). With their growing presence and importance in various organizations, managing call center operations more efficiently is of significant economic interest.

For most call centers, 60-70\% of operating expenses are capacity costs, and in particular human resource costs (Gans et al. 2003). Effective management of a call center requires call center managers to match up call center resources with workload. To forecast workload accurately, the first and critical step is to provide an accurate forecast of future call volumes. 


\section{Accepted by Manufacturing and Service Operations Management}

Usually two kinds of forecasts are needed by a call center manager for staffing and scheduling purposes: 1. Forecast the call volumes several days or weeks ahead; 2. On a particular day, dynamically update the forecast using newly available information as additional calls arrive throughout the day.

There are empirical justifications for the within-day updating, as both Avramidis et al. (2004) and Steckley et al. (2004) report evidence of positive correlation among time periods within a given day, i.e. intraday dependence. This updating is operationally both beneficial and feasible. We show that, if the within-day updating can be done appropriately, one can reduce the forecast error for the rest of the day substantially. Furthermore, one can perform the updating as early as a couple of hours into the working day to have a really significant effect. In turn, operational benefits would follow from the ability to change agent schedules according to the updated forecast. For example, to adjust for a revised forecast, call center managers may send people home early or have them take training sessions or work on alternative tasks; or they may arrange agents to work overtime or call in part-time or work-from-home agents. To the best of our knowledge, Weinberg et al. (2007) seems to be the only existing work dealing with forecast updating in the call center context.

Quantitative approaches to call arrival forecasting make use of historical call arrival data, which record the times at which calls arrive to the center. Typically call arrival data are aggregated into total numbers of arrivals during short time periods such as 15-minute or 30-minute intervals and subsequently the target of forecasting is future call volumes over such periods (Jongbloed and Koole 2001). We refer to the collection of such aggregated call volumes for a given day as the intraday call volume profile of that day (Section 3.1).

Early work on forecasting call arrivals usually ignores intraday dependence, and focuses on interday dependence among daily total volumes, partly due to lack of relevant data. Time series ARIMA models are usually called upon to perform the forecasting (Andrews and Cunningham 1995, Bianchi et al. 1993, 1998). Recently, with the availability of call-by-call data, stochastic models of call arrivals that take into account the intraday dependence have been introduced. Avramidis et al. (2004) develop doubly stochastic models that reproduce three empirically observed features of call arrival data, overdispersion, time-varying rate and intraday dependence. Brown et al. (2005) consider a time-varying Poisson process with random rate, and suggest a multiplicative two-way mixed-effects model to forecast future rate. Considering both interday forecasting and intraday updating, Weinberg et al. (2007) extend the work of Avramidis et al. and Brown et al. to model both interday and intraday depen- 
dences. A two-way multiplicative Bayesian Gaussian model is proposed, and a Markov chain Monte Carlo (MCMC) algorithm is developed for parameter estimation and forecasting; see Section 4.2 for details of the model. The Bayesian approach is theoretically very interesting, but the computation (MCMC) algorithm is rather sophisticated to implement and can take a long time to converge.

Intraday schedule updating has been investigated in the literature under the name of real-time work schedule adjustment for various service systems other than call centers. The real-time schedule adjustment process usually involves two phases (Hur et al. 2004). During the first phase, forecast monitoring systems (Hur 2002) are applied to detect when a forecast updating is needed; once an updating is determined as necessary, the forecast can be updated using a partially known demand forecasting model (Kekre et al. 1990, Guerrero and Elizondo 1997). The second phase involves schedule adjustment to handle the updated forecast, which has received very little attention until recently. For example, Thompson (1996) investigates various options for adjusting intraday schedules in the context of hospitality workforce management. Hur et al. (2004) propose schedule updating techniques, which are illustrated in the context of quick service restaurants. Easton and Goodale (2005) model absence recovery problems for service operations. In the context of call centers, Mehrotra et al. (2006) study in details intraday rescheduling of agent schedules and demonstrate its value.

The aforementioned forecasting approaches are mostly model-driven, where stochastic models are carefully designed to fit real world problems. In this paper, we introduce a system of statistical methods that can generate both interday (or day-to-day) and especially dynamic intraday (or within-day) forecasts. Unlike the model-driven approaches, our approach is data-driven, which starts with a dimension reduction technique to let the data suggest parsimonious underlying important features, and then uses them to build time series forecasting models. We illustrate that it can work as a competitive and complementary alternative to the model-driven approaches.

The data-driven nature suggests that our approach is robust (Section 4.3) and can work for a wide range of applications, for example, electricity demand forecasting (Cottet and Smith 2003) and bond yield curve forecasting (Diebold and Li 2006), to which it is of interest to apply our approach and see how it performs. Furthermore, our method can be easily implemented in widely available softwares such as MATLAB and SPLUS/R, and it computes faster than the Bayesian method of Weinberg et al. (2007), which makes it more suitable for real time forecast updating. In spite of its simplicity, our method is still very 
competitive in generating accurate forecasts (Section 4.5).

Our approach treats the intraday call volume profiles as a vector time series across days, where each intraday profile is considered as one observation of the vector time series. Thus, the dimensionality of the time series is high. We propose to first reduce the dimensionality by applying singular value decomposition (SVD) to the matrix of the intraday profiles. As a result, the high dimensional intraday profile series is summarized by a few pairs of interday and intraday features, (see (1) in Section 3.1). Then the intraday features are kept fixed, while the interday feature series are subject to time series modeling. Because the interday feature series are orthogonal, we can apply univariate time series forecasting techniques to forecast them separately. The resulting forecasts are subsequently combined with the intraday features to produce day-to-day forecasts of future intraday profiles. Distributional forecasts are also developed using a bootstrap procedure. Usually only a small number of interday and intraday feature pairs is needed. Two or three such pairs are sufficient to achieve very good forecasting performance for the two case studies in Section 4.

Our procedure for intraday dynamic updating is based on a technique known as penalized least squares. For a particular day, suppose we have obtained some day-to-day time series forecasts for the interday features. As calls arrive when the day progresses, one direct approach to update the time series forecasts is via a least squares regression using the newly available call volume profile as the response and the corresponding part of the intraday feature vectors as the independent variables, (see (6) in Section 3.3.1). The corresponding regression coefficients then give the updated interday feature forecasts. However, this approach relies solely on the new arrival information of the present day, and makes no use of the time series forecasts. Our penalized least squares method adjusts the direct approach by pushing the least squares updates towards the time series forecasts and finding a balance point between them, (see (8) in Section 3.3.2). This method effectively combines the newly available information with the information up to the end of the previous day. The empirical results in Section 4 show that such combination of information is very useful in reducing forecasting errors.

The rest of the paper is structured as follows. Section 2 introduces the call center arrival data that motivate the current research. Our forecasting approach is described in detail in Section 3. Section 3.1 introduces dimensionality reduction through SVD, and it is followed by Section 3.2 on the method for one- or multi-day-ahead intraday profile forecasting, and Section 3.3 on dynamic intraday updating. Both point and distributional forecasts are 
considered. Section 4 presents comparative studies of several competing methods based on their out-of-sample forecasting performance. Section 4.1 gives the forecasting performance measures we use and Section 4.2 describes the competing methods. Two simulated examples are presented in Section 4.3 to illustrate the robustness of our method. The data described in Section 2 are used in Section 4.4 to compare our method with several alternatives. In Section 4.5, a comparison between our method and the Bayesian approach of Weinberg et al. (2007) is provided using their call center data. We conclude in Section 5. Some technical details are relegated to the appendix.

\section{The Data}

The motivating data were gathered at an inbound call center of a major northeastern US financial firm between January 1 and October 26, 2003. The original database has detailed information about every call that got connected to this call center during this period (except three days when the data collecting equipment went out of order). The center is normally open between 7:00AM and midnight. For the current study, we are interested in understanding the arrival pattern of calls to the service queue and generating out-of-sample forecasts of future call volumes. As a result, the portion of the data of interest to us involves the information about the time every call arrives to the service queue.

The call arrival pattern is very different between weekdays and weekends. For this reason, we restrict our attention to weekdays. In particular, we focus on the 42 whole 5-day weeks between January 6 and October 24. There are some obvious abnormal days in the data, six holidays with very low call volumes and four days with no data; hence we decide to exclude them from our analysis. For a particular day, we divide the 17-hour operating period into sixty-eight 15-minute intervals, and record the number of calls arriving to the service queue during each interval. The aggregated data form a $200 \times 68$ count matrix, with each row corresponding to a particular day in the 42 weeks considered, and each column corresponding to one specific 15-minute interval between 7:00AM and midnight. The data are available from the authors upon request.

We found by plotting the data that call volumes almost always occur in predictable, repeating patterns. For example, it is typical that there is a peak around 11:00AM followed by a second, lower peak around 2:00PM. As an illustration, Figure 1(a) plots the average intraday arrival patterns for different weekdays. Different days of the week have different call 
arrival patterns. As shown in Figure 1(a), Fridays have the largest volumes in the morning and the smallest volumes after 17:00PM; while the opposite holds for Mondays. In addition, Figure 1(b) shows the intraday arrival patterns for the five days in the final week of the data set. The regularity of arrival patterns serves as the basis of any forecasting method.

Figure 1(b) also exhibits unusual arrival pattern for the Tuesday, during which there are too many calls arriving after 3:30PM. We shall keep such abnormal days for the case study to be reported in Section 4.4. Alternatively, we have tried to identify anomalous days using the technique in Shen and Huang (2005), and their call arrival profiles were then replaced by the average of the corresponding periods (i.e. same weekday and time-of-day) in the two adjacent weeks. This alternative treatment leads to similar forecasting comparison results (not shown) as those reported in Section 4.4.

Our data appear to possess heteroscedasticity (i.e., nonconstant variance) and overdispersion (i.e., variance greater than mean) (Avramidis et al. 2004), as shown in Figure 1(c). To stabilize the variance, we employed the root-unroot method (Brown et al., 2005) as fol-

lows. Let $N$ denote the call volume for a certain time interval. Set $X=\sqrt{N+1 / 4}$. Denote the forecast of $X$ by $\widehat{X}$. Then the forecast of $N$ is given by $\widehat{N}=\widehat{X}^{2}-1 / 4$. The motivation of the method is the following. It is a good approximation to assume that the call volume $N$ over a short time interval follows a Poisson distribution and, if $N \sim \operatorname{Poisson}(\lambda)$, then $X$ has approximately a mean of $\sqrt{\lambda}$ and a variance of $1 / 4$.

The intraday dependence reported by Avramidis et al. (2004) and Steckley et al. (2004) also exists in our data. For different weekdays, Figure 1(d) plots the intraday correlation between past call demand and future demand as a function of the observation time. The demand is calculated based on the square-root-transformed call volumes as motivated above. The intraday correlation appears to be significantly positive for most of the time, which empirically supports the potential effectiveness of intraday updating.

\section{Forecasting Methods}

\subsection{Dimension Reduction via Singular Value Decomposition}

Let $\mathbf{X}=\left(x_{i j}\right)$ be an $n \times m$ matrix that records the call volumes (or a transformation of the volumes as described in Section 2) for $n$ days with each day having $m$ time periods. The rows and columns of $\mathbf{X}$ correspond respectively to days and time periods within a day. The $i$ th row of $\mathbf{X}$, denoted as $\mathbf{x}_{i}^{T}=\left(x_{i 1}, \ldots, x_{i m}\right)$, is referred to as the intraday call volume 
Figure 1: Exploratory plots suggesting that both predictive regularity and abnormality are present in call center arrival data. (a) Average call volume profiles for different weekdays; (b) Intraday arrival patterns during the final week of our data. (c) Standard deviation of call volumes for different weekdays; (d) Intraday correlation between past call demand and future demand for different weekdays.
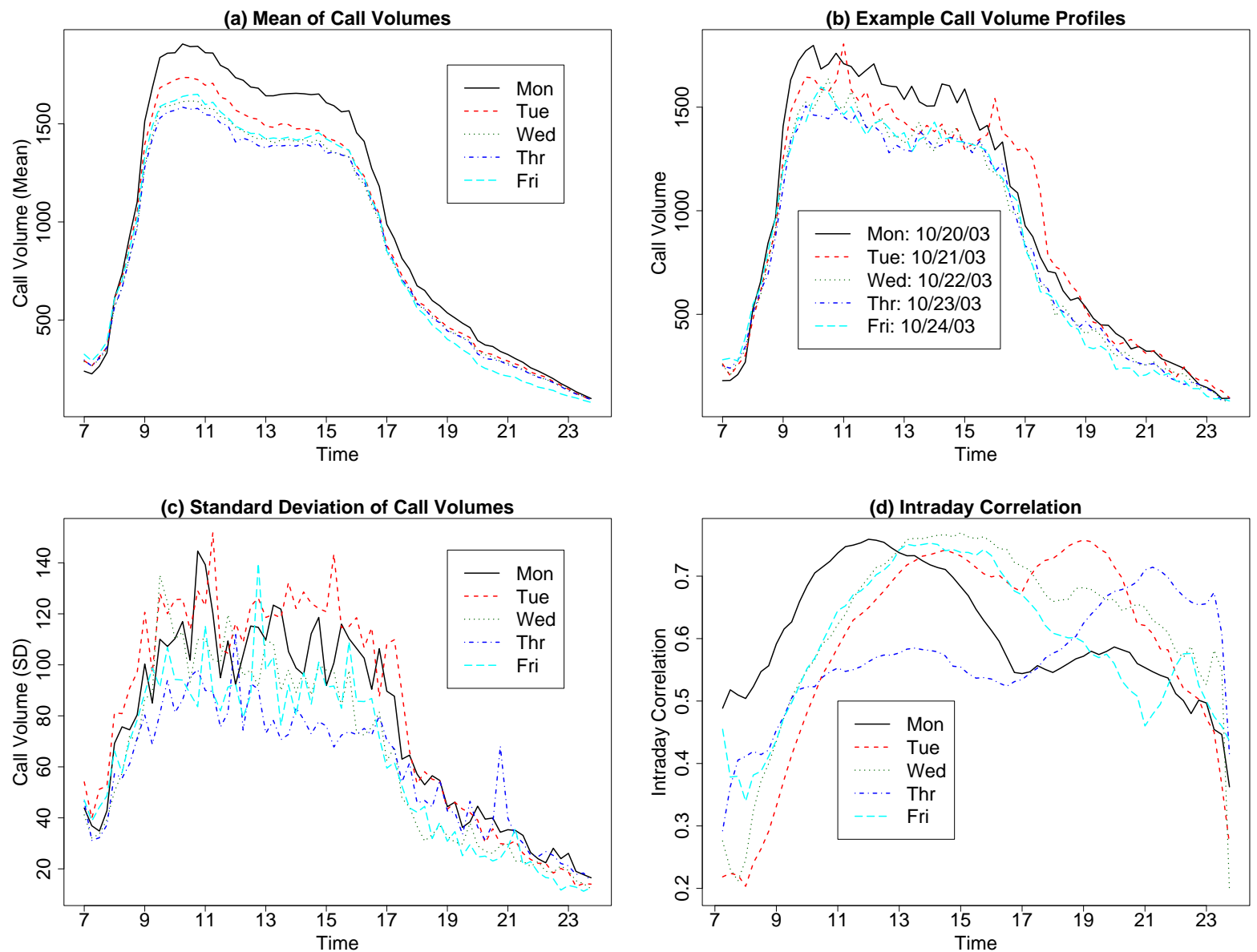
profile of the $i$ th day. The intraday profiles, $\mathbf{x}_{1}, \mathbf{x}_{2}, \ldots$, form a vector-valued time series taking values in $\mathbb{R}^{m}$. We want to build a time series model for this series and use it for forecasting. However, commonly used multivariate time series models such as VAR (Vector AutoRegressive models) and more general VARMA (Vector AutoRegressive and Moving Average models) (Reinsel 2003) are not directly applicable due to the large dimensionality of the time series we consider. For example, the dimensionality $m$ is 68 for our data and 169 for Weinberg's data.

Our approach starts from dimension reduction. We first seek a few basis vectors, denoted as $\mathbf{f}_{k}, k=1, \ldots, K$, such that all elements in the time series $\left\{\mathbf{x}_{i}\right\}$ can be represented (or approximated well) by these basis vectors. The number of the basis vectors $K$ should be much smaller than the dimensionality $m$ of the time series. Specifically, we consider the following decomposition,

$$
\mathbf{x}_{i}=\beta_{i 1} \mathbf{f}_{1}+\cdots+\beta_{i K} \mathbf{f}_{K}+\boldsymbol{\epsilon}_{i}, \quad i=1, \ldots, n,
$$

where $\mathbf{f}_{1}, \ldots, \mathbf{f}_{K} \in \mathbb{R}^{m}$ are the basis vectors and $\boldsymbol{\epsilon}_{1}, \ldots, \boldsymbol{\epsilon}_{n} \in \mathbb{R}^{m}$ are the error terms. We expect that the main features of $\mathbf{x}_{i}$ can be summarized by a linear combination of the basis vectors so that the error terms in (1) would be small in magnitude. This can be achieved by solving the following minimization problem for fixed $K$,

$$
\min _{\substack{\beta_{i 1}, \ldots, \beta_{i K} \\ \mathbf{f}_{1}, \ldots, \mathbf{f}_{K}}} \sum_{i=1}^{n}\left\|\boldsymbol{\epsilon}_{i}\right\|^{2}=\min _{\substack{\beta_{i 1}, \ldots, \beta_{i K} \\ \mathbf{f}_{1}, \ldots, \mathbf{f}_{K}}} \sum_{i=1}^{n}\left\|\mathbf{x}_{i}-\left(\beta_{i 1} \mathbf{f}_{1}+\cdots+\beta_{i K} \mathbf{f}_{K}\right)\right\|^{2} .
$$

For identifiability, we require in (2) that $\mathbf{f}_{i}^{T} \mathbf{f}_{j}=\delta_{i j}$, where $\delta_{i j}$ is the Kronecker delta which equals 1 for $i=j$ and 0 otherwise. The solution to this problem is actually given by the singular value decomposition (SVD) of the matrix $\mathbf{X}$ as shown below.

The SVD of the matrix $\mathbf{X}$ can be expressed as

$$
\mathbf{X}=\mathbf{U S V}^{T}
$$

where $\mathbf{U}$ is an $n \times m$ matrix with orthonormal columns, $\mathbf{S}$ is an $m \times m$ diagonal matrix, and $\mathbf{V}$ is an $m \times m$ orthogonal matrix. The columns of $\mathbf{U},\left\{\mathbf{u}_{k}=\left(u_{1 k}, \ldots, u_{n k}\right)^{T}\right\}$, namely the left singular vectors, satisfy $\mathbf{u}_{i}^{T} \mathbf{u}_{j}=\delta_{i j}$. The columns of $\mathbf{V},\left\{\mathbf{v}_{k}=\left(v_{1 k}, \ldots, v_{m k}\right)^{T}\right\}$, or the right singular vectors, satisfy $\mathbf{v}_{i}^{T} \mathbf{v}_{j}=\delta_{i j}$. The diagonal elements of $\mathbf{S}$ are the singular values, which are usually ordered decreasingly. Let $\mathbf{S}=\operatorname{diag}\left(s_{1}, \ldots, s_{m}\right)$ and $r=\operatorname{rank}(\mathbf{X})$. Then $s_{1} \geq s_{2} \geq \cdots \geq s_{r}>0$, and $s_{k}=0$ for $r+1 \leq k \leq m$. 
It then follows from (3) that

$$
\mathbf{x}_{i}=s_{1} u_{i 1} \mathbf{v}_{1}+\cdots+s_{r} u_{i r} \mathbf{v}_{r}
$$

Keeping only the terms associated with the largest $K$ singular values, we have the following approximation,

$$
\mathbf{x}_{i} \simeq\left(s_{1} u_{i 1}\right) \mathbf{v}_{1}+\cdots+\left(s_{K} u_{i K}\right) \mathbf{v}_{K}
$$

This $K$-term approximation is an optimal solution for the minimization problem (2). More precisely, $\beta_{i k}=s_{k} u_{i k}$ and $\mathbf{f}_{k}=\mathbf{v}_{k}, i=1, \ldots, n, k=1, \ldots, K$, solve (2), and the solution is unique up to a sign change to $\mathbf{f}_{k}$ (Eckart and Young 1936). Thus, we have formally obtained the decomposition (1) using the SVD of $\mathbf{X}$. Note that requiring $\mathbf{f}_{i}^{T} \mathbf{f}_{j}=\delta_{i j}$ is only one way to make the solution to (2) unique. Alternatively, if we require $\sum_{i=1}^{n} \beta_{i k} \beta_{i l}=\delta_{k l}$, then one solution to (2) will be $\beta_{i k}=u_{i k}$ and $\mathbf{f}_{k}=s_{k} \mathbf{v}_{k}$, which is unique up to a sign change to $\mathbf{f}_{k}$.

Given a historical data matrix $\mathbf{X}$, to estimate the model (1), we apply SVD to $\mathbf{X}$ and extract the first $K$ pairs of singular vectors, along with the corresponding singular values, which then lead to the intraday feature vectors $\mathbf{f}_{1}, \ldots, \mathbf{f}_{K}$, and the interday feature series $\left\{\beta_{i 1}\right\}, \ldots,\left\{\beta_{i K}\right\}$. Such features will be used as the basis of our forecasting methods as described below in Sections 3.2 and 3.3. From the decomposition (1), the high-dimensional intraday profiles $\mathbf{x}_{i}$ are concisely summarized by a small number of interday feature series, using the same number of intraday feature vectors as the bases. The decreasing order among the singular values suggests that the feature series/vectors are naturally ordered according to importance in approximating the data matrix. By using a small $K$, we achieve a substantial dimension reduction - from $m$ to $K$. In the real data example in Section 4.4, a $K$ of 2 or 3 already gives good forecasting results, while $m$ is 68 . One attractive feature of the decomposition (1) is that it effectively separates out the intraday and interday variations, both of which are present in the intraday profile time series. Another benefit is that the variations are decomposed into orthogonal components, which greatly simplifies our forecasting model as described below in Section 3.2.

Our SVD based dimension reduction is closely related to principal components analysis (PCA) when principal components (PCs) are calculated from the covariance matrix (Jolliffe 2002). If the data matrix $\mathbf{X}$ is column centered such that the columns (viewed as variables) have mean zero, then $\mathbf{X}^{T} \mathbf{X}$ is proportional to the sample covariance matrix of the columns of $\mathbf{X}$. According to (3), $\mathbf{X}^{T} \mathbf{X}=\mathbf{V S}^{2} \mathbf{V}^{T}$, which means that the columns $\mathbf{v}_{k}$ of $\mathbf{V}$, or the intraday feature vectors $\mathbf{f}_{k}$, are indeed the PC loading vectors, or $\mathbf{X} \mathbf{v}_{k}$ are the PCs; and the 
squared singular values $s_{k}^{2}$ are proportional to the variances of the PCs. In PCA, the quantity $s_{k}^{2} / \sum_{i} s_{i}^{2}$ measures the relative importance of the $k$ th PC, and can be plotted versus $k$ in a scree plot. To decide on the number of PCs, one usually looks for an "elbow" in the scree plot, formed by a steep drop followed by a relatively flat tail of small values. The number of PCs needed then corresponds to the integer prior to the elbow. Although we do not center columns in our context, as there is no good reason for doing it, the connection with PCA does suggest the scree plot as a possible way to pick the number of important features $K$ (Shen and Huang 2005). In practice, one can also choose $K$ by comparing out-of-sample forecasting performance using historical data for different choices.

\subsection{Interday Forecasting}

Consider forecasting the intraday call volume profile $\mathbf{x}_{n+h}(h>0)$ using the historical data $\mathbf{x}_{1}, \ldots, \mathbf{x}_{n}$. By making use of the model (1), forecasting a $m$-dimensional time series $\left\{\mathbf{x}_{i}\right\}$ reduces to forecasting $K$ one-dimensional interday feature series $\left\{\beta_{i 1}\right\}, \ldots,\left\{\beta_{i K}\right\}$. We consider the decomposition (1) with the $\mathbf{f}_{k}$ 's replaced by the ones extracted using SVD from the historical data matrix $\mathbf{X}$.

\subsubsection{Point Forecast}

If we can obtain a forecast of the coefficients $\beta_{n+h, 1}, \ldots, \beta_{n+h, K}$, then a forecast of $\mathbf{x}_{n+h}$ is given by

$$
\hat{\mathbf{x}}_{n+h}=\hat{\beta}_{n+h, 1} \mathbf{f}_{1}+\cdots+\hat{\beta}_{n+h, K} \mathbf{f}_{K},
$$

where $\hat{\beta}_{n+h, k}$ is a forecast of $\beta_{n+h, k}, k=1, \ldots, K$. Denote $\mathbf{f}_{k}=\left(f_{1 k}, \ldots, f_{m k}\right)^{T}$. The forecast of the call volume of the $j$ th time period of day $n+h$ is then given by

$$
\hat{x}_{n+h, j}=\hat{\beta}_{n+h, 1} f_{j 1}+\cdots+\hat{\beta}_{n+h, K} f_{j K}, \quad j=1, \ldots, m .
$$

Because of the way the SVD is constructed, $\left(\beta_{1 k}, \ldots, \beta_{n k}\right)$ is orthogonal to $\left(\beta_{1 l}, \ldots, \beta_{n l}\right)$ for $k \neq l$. This lack of contemporaneous correlation suggests that the cross-correlations at non-zero lags are likely to be small. Therefore it is reasonable to believe that it is adequate to forecast each series $\left\{\beta_{i k}\right\}$ separately using univariate time series methods, for $k=1, \ldots, K$. To forecast each series, one could use univariate time series models such as exponential smoothing, ARIMA models (Box et al. 1994), state space models (Harvey 1990) and other suitable models. Which model to use would depend on the actual situation, and can be decided on by analyzing historical data. 
In our case study of the call center data described in Section 2, we apply a varying coefficient AR(1) model that takes into account the day-of-the-week effect. This model works well in our out-of-sample forecasting exercise in Section 4.4.

\subsubsection{Distributional Forecast}

For the agent scheduling and updating problem discussed in Section 1, call center managers also need distributional forecasts of $\mathbf{x}_{n+h}$, such as prediction intervals and densities, in addition to point forecasts. Understanding the variability around point forecasts is essential for several reasons. A threshold on predicted volumes could be determined to trigger appropriate managerial actions (Jongbloed and Koole 2001). The staffing level for a time period depends on both the mean and the variability of the forecast. As suggested by Steckley et al. (2004), the higher the variability, the higher the staffing targets should be in order to achieve certain service level goals. In addition, the variability in the forecasts may provide motivation for contracting additional contingency resources, routing calls across different sites, and cross-training across different queues (Betts et al. 2000, Wallace and Whitt 2005).

As our approach assumes no probabilistic models, we propose to use the following nonparametric bootstrap method to derive the distributional forecasts. Bootstrapping is an increasingly popular method for estimating the sampling distribution of an estimator by sampling with replacement from the original sample (Efron and Tibshirani 1994).

For a fixed $k$, using the fitted time series model for the series $\left\{\beta_{i k}\right\}$ recursively, we can generate $B$ series $\left\{\hat{\beta}_{n+1, k}^{b}, \ldots, \hat{\beta}_{n+h, k}^{b}\right\}, 1 \leq b \leq B$, by bootstrapping the model errors from the fitted time series model (see Appendix A.1 for details). In our data examples (Section 4), we set the number of bootstrap samples $B$ to be 1000. Similarly, we can bootstrap the approximation error profile $\boldsymbol{\epsilon}$ in the model (1) from the fitted error profiles $\hat{\boldsymbol{\epsilon}}_{1}, \ldots, \hat{\boldsymbol{\epsilon}}_{n}$ obtained by fitting the model (1) to the historical $n$ call volume profiles. Let $\hat{\boldsymbol{\epsilon}}^{b}, 1 \leq b \leq B$, denote a random draw with replacement from $\hat{\boldsymbol{\epsilon}}_{1}, \ldots, \hat{\boldsymbol{\epsilon}}_{n}$. Then we obtain $B$ forecasts of $\mathbf{x}_{n+h}$,

$$
\widehat{\mathbf{x}}_{n+h}^{b}=\hat{\beta}_{n+h, 1}^{b} \mathbf{f}_{1}+\cdots+\hat{\beta}_{n+h, K}^{b} \mathbf{f}_{K}+\hat{\boldsymbol{\epsilon}}^{b}, \quad b=1, \ldots, B,
$$

from which interval and density forecasts can be derived. For a 100(1- $\alpha) \%$ forecast interval, the two end points are correspondingly the $\alpha / 2$ and $(1-\alpha) / 2$ empirical percentiles of $\left\{\widehat{\mathbf{x}}_{n+h}^{1}, \ldots, \widehat{\mathbf{x}}_{n+h}^{B}\right\}$. The histogram or density estimate of $\left\{\widehat{\mathbf{x}}_{n+h}^{1}, \ldots, \widehat{\mathbf{x}}_{n+h}^{B}\right\}$ then gives a density forecast of $\mathbf{x}_{n+h}$. 
Our approach makes several implicit assumptions. First of all, the time series model employed should be a reasonably good approximation to reality. Second, for the validity of the nonparametric bootstrap, the time series model errors are assumed to be independent and identically distributed with mean zero. This is the reason why the approximation error profile $\boldsymbol{\epsilon}$ is bootstrapped as a whole, so that we can capture any remaining intraday correlation. These implicit assumptions remain applicable when we discuss dynamic intraday updating in the next subsection. Note that our approach does not assume a specific parametric distribution for the errors.

\subsection{Dynamic Intraday Updating}

Using the method in the previous section, a manager is able to forecast the call volume profile for day $n+h$ at the end of day $n$. In particular, $h=1$ corresponds to the next day. As calls arrive during day $n+1$, the manager may want to dynamically update her forecast for the remainder of the day using the information from the early part of that day. This dynamic updating is useful because it adds flexibility to her allocation of available resources, which then leads to higher efficiency and productivity, as well as better quality of service. By reevaluating her forecast for the remainder of the day, she can then schedule meetings or training sessions for agents free from work at short-notice, or call in back-up agents.

Suppose we have available the call volumes during the first $m_{0}$ time periods of day $n+1$. Denote them collectively as $\mathbf{x}_{n+1}^{e}=\left(x_{n+1,1}, \ldots, x_{n+1, m_{0}}\right)^{T}$, a vector containing the first $m_{0}$ elements of $\mathbf{x}_{n+1}$. Denote $\mathbf{x}_{n+1}^{l}=\left(x_{n+1, m_{0}+1}, \ldots, x_{n+1, m}\right)^{T}$ to be the intraday call volume profile for the later part of day $n+1$. For notational simplicity, we suppress the dependence of $\mathbf{x}_{n+1}^{e}$ and $\mathbf{x}_{n+1}^{l}$ on $m_{0}$.

Let $\hat{\beta}_{n+1, k}^{\mathrm{TS}}$ be a time series (TS) forecast based on $\left\{\beta_{i, k}\right\}$, for $k=1, \ldots, K$, using information up to the end of day $n$. The TS forecast of $\mathbf{x}_{n+1}^{l}$ is then given by

$$
\hat{x}_{n+1, j}^{\mathrm{TS}}=\hat{\beta}_{n+1,1}^{\mathrm{TS}} f_{j 1}+\cdots+\hat{\beta}_{n+1, K}^{\mathrm{TS}} f_{j K}, \quad j=m_{0}+1, \ldots, m .
$$

These forecasts do not utilize any new information of day $n+1$. Below, we discuss two ways to incorporate the new information in $\mathbf{x}_{n+1}^{e}$ to obtain an updated forecast of $\mathbf{x}_{n+1}^{l}$. 


\subsubsection{Direct Least Squares Updating}

When being applied to the intraday profile of day $n+1$, the decomposition (1) can be written as

$$
x_{n+1, j}=\beta_{n+1,1} f_{j 1}+\cdots+\beta_{n+1, K} f_{j K}+\epsilon_{n+1, j}, \quad j=1, \ldots, m .
$$

Let $\mathbf{F}^{e}$ be a $m_{0} \times K$ matrix whose $(j, k)$ th entry is $f_{j k}, 1 \leq j \leq m_{0}, 1 \leq k \leq K, \boldsymbol{\beta}_{n+1}=$ $\left(\beta_{n+1,1}, \ldots, \beta_{n+1, K}\right)^{T}$, and $\boldsymbol{\epsilon}_{n+1}^{e}=\left(\epsilon_{n+1,1}, \ldots, \epsilon_{n+1, m_{0}}\right)^{T}$. Then, with the availability of $\mathbf{x}_{n+1}^{e}$, we have the following linear regression model,

$$
\mathbf{x}_{n+1}^{e}=\mathbf{F}^{e} \boldsymbol{\beta}_{n+1}+\boldsymbol{\epsilon}_{n+1}^{e} .
$$

This suggests that we can forecast $\boldsymbol{\beta}_{n+1}$ by the method of least squares (LS). Solving

$$
\min _{\boldsymbol{\beta}_{n+1}}\left\|\mathbf{x}_{n+1}^{e}-\mathbf{F}^{e} \boldsymbol{\beta}_{n+1}\right\|^{2}
$$

we obtain that

$$
\hat{\boldsymbol{\beta}}_{n+1}^{\mathrm{LS}}=\left(\mathbf{F}^{e T} \mathbf{F}^{e}\right)^{-1} \mathbf{F}^{e T} \mathbf{x}_{n+1}^{e}
$$

The LS forecast of $\mathbf{x}_{n+1}^{l}$ is then given by

$$
\hat{x}_{n+1, j}^{\mathrm{LS}}=\hat{\beta}_{n+1,1}^{\mathrm{LS}} f_{j 1}+\cdots+\hat{\beta}_{n+1, K}^{\mathrm{LS}} f_{j K}, \quad j=m_{0}+1, \ldots, m .
$$

To make the LS forecast of $\boldsymbol{\beta}_{n+1}$ uniquely defined, we require that $m_{0} \geq K$. This makes sense intuitively in that one needs more observations than parameters, in order to carry out the least squares estimation. Operationally, this suggests a trade-off between having more feature factors (i.e. a larger $K$ ) and updating forecasts earlier in the day (a smaller $m_{0}$ ). One achieves a better approximation of the data matrix $\mathbf{X}$ by having more factors; however, the manager then has to wait longer to start her direct LS forecast updating. Below we propose a penalized LS updating approach which seems to offer a solution to this trade-off.

\subsubsection{Penalized Least Squares Updating}

Direct least squares updating makes use of the additional information available at the early part of day $n+1$, but it needs a sufficient amount of data (i.e. a large enough $m_{0}$ ) in order for $\hat{\boldsymbol{\beta}}_{n+1}^{\mathrm{LS}}$ to be reliable. This might create a problem if the manager wants to update her forecast early in the morning, for example, at 8:00AM with $m_{0}=4$ or 10:00AM with $m_{0}=12$ for the data in Section 2. Another disadvantage of the direct LS updating is that it does not make 
full use of the historical information other than the estimated intraday feature vectors. In particular, it ignores the day-to-day dependence present in the interday feature series.

We propose combining the least squares forecast with the time series forecast of $\boldsymbol{\beta}_{n+1}$ by using the idea of penalization. Specifically, we minimize with respect to $\beta_{n+1,1}, \ldots, \beta_{n+1, K}$ the following penalized least squares (PLS) criterion,

$$
\sum_{j=1}^{m_{0}}\left|x_{n+1, j}-\left(\beta_{n+1,1} f_{j 1}+\cdots+\beta_{n+1, K} f_{j K}\right)\right|^{2}+\lambda \sum_{k=1}^{K}\left|\beta_{n+1, k}-\hat{\beta}_{n+1, k}^{\mathrm{TS}}\right|^{2},
$$

where $\hat{\beta}_{n+1, k}^{\text {TS }}$ is a time series forecast based on the information up to the end of day $n$, and $\lambda>0$ is a penalty parameter. Since only one penalty parameter is used, it makes sense for the $K$ time series $\left\{\beta_{i k}\right\}, 1 \leq k \leq K$, to be roughly on the same scale. This can be achieved by requiring $\sum_{i=1}^{n} \beta_{i k} \beta_{i l}=\delta_{k l}$ or $(1 / n) \sum_{i=1}^{n} \beta_{i k} \beta_{i l}=\delta_{k l}$ in $(2)$.

The PLS criterion (8) involves two terms: the first term measures how well the model prediction matches the observed call volumes in the early part of the day, while the second term penalizes a large departure from the time series forecast. The $\boldsymbol{\beta}_{n+1}$ obtained as the solution to the minimization problem will be a compromise between the two terms based on the size of $\lambda$, the penalty parameter. In practice, $\lambda$ can be selected based on the forecasting performance on a rolling hold-out sample; see Section 4.4.2 for a detailed description of one selection procedure. The case study in Section 4.4.2 also exemplifies how one can understand the relative importance of the two terms.

For each given $\lambda$, the PLS problem can be rewritten in a constrained optimization form:

$$
\begin{aligned}
& \operatorname{minimize} \sum_{k=1}^{K}\left|\beta_{n+1, k}-\hat{\beta}_{n+1, k}^{\mathrm{TS}}\right|^{2} \text { subject to } \\
& \sum_{j=1}^{m_{0}}\left|x_{n+1, j}-\left(\beta_{n+1,1} f_{j 1}+\cdots+\beta_{n+1, K} f_{j K}\right)\right|^{2} \leq \xi,
\end{aligned}
$$

where $\xi=\xi(\lambda)$ is chosen appropriately so that the solution to this problem and the previous one are the same for the given $\lambda$. The optimization problem can then be interpreted as follows, among all the $\boldsymbol{\beta}_{n+1}$ 's that yield forecasts which adequately match the observed call volumes of the early part of the day, find the one that is closest to the time series forecast.

We can express the PLS criterion (8) in the following matrix form,

$$
\left(\mathbf{x}_{n+1}^{e}-\mathbf{F}^{e} \boldsymbol{\beta}_{n+1}\right)^{T}\left(\mathbf{x}_{n+1}^{e}-\mathbf{F}^{e} \boldsymbol{\beta}_{n+1}\right)+\lambda\left(\boldsymbol{\beta}_{n+1}-\hat{\boldsymbol{\beta}}_{n+1}^{\mathrm{TS}}\right)^{T}\left(\boldsymbol{\beta}_{n+1}-\hat{\boldsymbol{\beta}}_{n+1}^{\mathrm{TS}}\right) .
$$


Minimizing this criterion gives us the PLS forecast of $\boldsymbol{\beta}_{n+1}$ as

$$
\hat{\boldsymbol{\beta}}_{n+1}^{\mathrm{PLS}}=\left(\mathbf{F}^{e T} \mathbf{F}^{e}+\lambda \mathbf{I}\right)^{-1}\left(\mathbf{F}^{e T} \mathbf{x}_{n+1}^{e}+\lambda \hat{\boldsymbol{\beta}}_{n+1}^{\mathrm{TS}}\right) .
$$

The PLS forecast of $\mathbf{x}_{n+1}^{l}$ is then given by,

$$
\hat{x}_{n+1, j}^{\mathrm{PLS}}=\hat{\beta}_{n+1,1}^{\mathrm{PLS}} f_{j 1}+\cdots+\hat{\beta}_{n+1, K}^{\mathrm{PLS}} f_{j K}, \quad j=m_{0}+1, \ldots, m .
$$

The following Theorem 1 suggests that the PLS forecast $\hat{\boldsymbol{\beta}}_{n+1}^{\text {PLS }}$ is actually a weighted average of the LS forecast and the TS forecast with the weights controlled by the penalty parameter $\lambda$. Note that, if $\lambda=0, \hat{\boldsymbol{\beta}}_{n+1}^{\mathrm{PLS}}$ is simply $\hat{\boldsymbol{\beta}}_{n+1}^{\mathrm{LS}}$; if $\lambda \rightarrow \infty$, then $\hat{\boldsymbol{\beta}}_{n+1}^{\mathrm{PLS}}$ reduces to $\hat{\boldsymbol{\beta}}_{n+1}^{\mathrm{TS}}$.

\section{Theorem 1}

$$
\hat{\boldsymbol{\beta}}_{n+1}^{\mathrm{PLS}}=A(\lambda) \hat{\boldsymbol{\beta}}_{n+1}^{\mathrm{LS}}+(I-A(\lambda)) \hat{\boldsymbol{\beta}}_{n+1}^{\mathrm{TS}},
$$

where $A(\lambda)$ is a $K \times K$ matrix satisfying $A(0)=I$ and $A(\lambda) \rightarrow 0$ as $\lambda \rightarrow \infty$.

We point out that the PLS idea can be derived from a Bayesian perspective. To this end, we treat the TS forecast $\hat{\boldsymbol{\beta}}_{n+1}^{\mathrm{TS}}$ as our prior knowledge of $\boldsymbol{\beta}_{n+1}$, and model $\beta_{n+1, k}(1 \leq k \leq K)$ as random draws from a "prior" normal distribution with mean $\hat{\beta}_{n+1, k}^{\text {TS }}$ and variance $\sigma_{0}^{2}$. The error term $\epsilon_{n+1, j}$ in the expression (6) is modeled as normal with mean 0 and variance $\sigma^{2}$. Then the "posterior" density function of $\boldsymbol{\beta}_{n+1}$ is maximized by the solution of the PLS problem (8) with $\lambda=\sigma^{2} / \sigma_{0}^{2}$.

There exists a connection between our PLS approach and the widely used ridge regression (Hoerl and Kennard 1970a,b) in regression analysis. Define $\widetilde{\mathbf{x}}_{n+1}^{e}=\mathbf{x}_{n+1}^{e}-\mathbf{F}^{e} \hat{\boldsymbol{\beta}}_{n+1}^{\mathrm{TS}}$ and $\widetilde{\boldsymbol{\beta}}_{n+1}=\boldsymbol{\beta}_{n+1}-\hat{\boldsymbol{\beta}}_{n+1}^{\mathrm{TS}}$. Consider a ridge regression with $\widetilde{\mathbf{x}}_{n+1}^{e}$ as the response, $\mathbf{F}^{e}$ as the design matrix, and $\widetilde{\boldsymbol{\beta}}_{n+1}$ as the regression coefficient vector. The ridge estimate of $\widetilde{\boldsymbol{\beta}}_{n+1}$ is

$$
\widehat{\widetilde{\boldsymbol{\beta}}}_{n+1}=\left(\mathbf{F}^{e T} \mathbf{F}^{e}+\lambda \mathbf{I}\right)^{-1} \mathbf{F}^{e T} \widetilde{\mathbf{x}}_{n+1}^{e},
$$

which minimizes the criterion

$$
\left(\widetilde{\mathbf{x}}_{n+1}^{e}-\mathbf{F}^{e} \widetilde{\boldsymbol{\beta}}_{n+1}\right)^{T}\left(\widetilde{\mathbf{x}}_{n+1}^{e}-\mathbf{F}^{e} \widetilde{\boldsymbol{\beta}}_{n+1}\right)+\lambda \widetilde{\boldsymbol{\beta}}_{n+1}^{T} \widetilde{\boldsymbol{\beta}}_{n+1} .
$$

This is the same as the PLS criterion in (9) up to a reparametrization $\widetilde{\boldsymbol{\beta}}_{n+1}=\boldsymbol{\beta}_{n+1}-\hat{\boldsymbol{\beta}}_{n+1}^{\mathrm{TS}}$ Therefore the minimizers of the two optimization problems are linked through $\widehat{\widetilde{\boldsymbol{\beta}}}_{n+1}=\hat{\boldsymbol{\beta}}_{n+1}^{\text {PLS }}$ $\hat{\boldsymbol{\beta}}_{n+1}^{\mathrm{TS}}$. The ridge estimate in (12) shrinks the corresponding least squares estimate toward 
0. Correspondingly, our PLS forecast modifies the least squares forecast $\hat{\boldsymbol{\beta}}^{\mathrm{LS}}$ by shrinking it towards the TS forecast $\hat{\boldsymbol{\beta}}^{\mathrm{TS}}$. As noticed by Hoerl and Kennard, the shrinkage of ridge regression has the beneficial effect of reduced variance. Similar variance reduction is expected for our PLS forecast.

\subsubsection{Distributional Forecast Updating}

The PLS provides a point forecast updating scheme. For the corresponding distributional updating of $\mathbf{x}_{n+1}^{l}$, we use a nonparametric bootstrap procedure similar to the one described in Section 3.2.2. First, by bootstrapping the errors from the time series forecasting models for $\boldsymbol{\beta}_{n+1}$, we obtain $B$ time series forecasts $\hat{\boldsymbol{\beta}}_{n+1}^{\mathrm{TS}, b}$ as discussed in Appendix A.1. These forecasts in turn lead to $B$ PLS forecasts $\hat{\boldsymbol{\beta}}_{n+1}^{\text {PLS }, b}$ according to (10).

Then, we derive $B$ PLS updates of $\mathbf{x}_{n+1}^{l}$ as follows,

$$
\hat{x}_{n+1, j}^{\mathrm{PLS}, b}=\hat{\beta}_{n+1,1}^{\mathrm{PLS}, b} f_{j 1}+\cdots+\hat{\beta}_{n+1, K}^{\mathrm{PLS}, b} f_{j K}+\hat{\epsilon}_{j}^{b}, \quad j=m_{0}+1, \ldots, m ; \quad b=1, \ldots, B,
$$

where $\hat{\boldsymbol{\epsilon}}^{b}=\left(\hat{\epsilon}_{1}^{b}, \ldots, \hat{\epsilon}_{m}^{b}\right)^{T}$ is sampled with replacement from the fitted error profiles $\hat{\boldsymbol{\epsilon}}_{1}, \ldots, \hat{\boldsymbol{\epsilon}}_{n}$ of the historical data. The interval and density forecasts of $x_{n+1, j}$ are then obtained using the empirical distribution of $\left\{\hat{x}_{n+1, j}^{\mathrm{PLS}, b}: 1 \leq b \leq B\right\}$. For a $100(1-\alpha) \%$ forecast interval, the two end points are the $\alpha / 2$ and $(1-\alpha) / 2$ empirical percentiles of $\left\{\hat{x}_{n+1, j}^{\mathrm{PLS}, b}\right\}$. The histogram or density estimate of $\left\{\hat{x}_{n+1, j}^{\mathrm{PLS}, b}\right\}$ then gives a density forecast of $x_{n+1, j}$.

\section{Forecasting Performance Comparison}

We conduct out-of-sample rolling forecasting exercises on simulated and real data sets to evaluate the proposed forecasting method and compare it with competing methods. Both one-day-ahead forecasting and dynamic intraday updating are considered. The root-unroot method (Section 2) is applied to all forecasting methods to deal with heteroscedasticity. The methods are described on the root-transformed data, while the unroot-transformation is needed to derive forecasts of the call volumes.

Section 4.1 presents several forecasting performance measures. Several competing methods are described in Section 4.2. Two simulated examples are used in Section 4.3 to compare our data-driven approach with two model based approaches. The two case studies are reported separately in Sections 4.4 and 4.5, using the data described in Section 2 and the data used in Weinberg et al. (2007). By showing the forecast-accuracy comparison on two datasets, it provides evidence of the robustness and general applicability of our approach. 


\subsection{Performance Measures}

Two measures are used for assessing and comparing point forecast performances. Let $N_{i j}$ denote the call volume on day $i$ during period $j$, and $\widehat{N}_{i j}$ be a forecast of $N_{i j}$. Suppose we are interested in forecasting the whole intraday profile for day $i$, as for example, in oneday-ahead forecasting. Then, the root mean squared error (RMSE) and mean relative error (MRE) are defined for day $i$ as follows,

$$
\operatorname{RMSE}_{i}=\sqrt{\frac{1}{m} \sum_{j=1}^{m}\left(\widehat{N}_{i j}-N_{i j}\right)^{2}} \text { and } \operatorname{MRE}_{i}=\frac{100}{m} \sum_{j=1}^{m} \frac{\left|\widehat{N}_{i j}-N_{i j}\right|}{N_{i j}} .
$$

We now discuss how to evaluate our bootstrap based forecast intervals. As described in Section 3.2, we first obtain a bootstrap sample of $B$ forecasts of $N_{i j}$. It is proposed to use $\left[\widehat{N}_{i j}^{100(\alpha / 2)}, \widehat{N}_{i j}^{100(1-\alpha / 2)}\right]$ as one $100(1-\alpha) \%$ forecast interval of $N_{i j}$, where $\widehat{N}_{i j}^{Q}$ is the $Q$ th quantile of the bootstrap sample. In practice, people commonly use $95 \%$ prediction intervals, which correspond to $\alpha=0.05$. For day $i$, we compute the $95 \%$ coverage probability (COVER) and the average forecast interval width (WIDTH) as follows

$$
\mathrm{COVER}_{i}=\frac{1}{m} \sum_{j=1}^{m} \mathrm{I}\left(\widehat{N}_{i j}^{2.5}<N_{i j}<\widehat{N}_{i j}^{97.5}\right) \quad \text { and } \quad \mathrm{WIDTH}_{i}=\frac{1}{m} \sum_{j=1}^{m}\left(\widehat{N}_{i j}^{97.5}-\widehat{N}_{i j}^{2.5}\right),
$$

with I being the indicator function. We expect the empirical coverage probability to be close to the nominal value, and the narrower the forecast interval, the better.

For dynamic updating, suppose we update our forecast after time period $j$, then the above measures can be calculated by averaging over only those remaining periods after time period $j$. Summary statistics (over days in the forecasting set) such as mean, median, quartiles of the measures can be reported to compare the performance of different forecasting methods.

\subsection{Competing Methods}

\subsubsection{Interday Forecasting}

The Benchmark. The first alternative is a simple method that is currently used in the call center industry (Cleveland and Mayben 2004, Weinberg et al. 2007), which incorporates both day-of-the-week and time-of-day effects in a linear additive model. Specifically, let $d_{i}$ denote the day-of-the-week of day $i$, and $x_{i j}=\sqrt{N_{i j}+1 / 4}$ with $N_{i j}$ being the call volume during period $j$ of day $i$. Then the model is

$$
x_{i j}=\mu+\alpha_{d_{i}}+\beta_{j}+\epsilon_{i j}, \quad \epsilon_{i j} \sim N\left(0, \sigma^{2}\right) .
$$


We refer to this approach as the historical average (HA) approach, because it essentially uses historical averages of the same day-of-the-week as a forecast for the current day's squareroot-transformed call volumes.

A Bayesian Gaussian Model. The second alternative is the Bayesian approach proposed in Weinberg et al. (2007), which assumes $N_{i j}$ is a Poisson random variable with a random rate that depends on both day-of-the-week and time-of-day. Using the root-unroot method, the following Bayesian Gaussian (BG) model is proposed,

$$
\left\{\begin{array}{l}
x_{i j}=g_{d_{i}}\left(t_{j}\right) y_{i}+\epsilon_{i j}, \quad \epsilon_{i j} \sim N\left(0, \sigma^{2}\right), \\
y_{i}-\alpha_{d_{i}}=\beta\left(y_{i-1}-\alpha_{d_{i-1}}\right)+\eta_{i}, \quad \eta_{i} \sim N\left(0, \phi^{2}\right), \\
\frac{d^{2} g_{d_{i}}\left(t_{j}\right)}{d t_{j}^{2}}=\tau_{d_{i}} \frac{d W_{d_{i}}\left(t_{j}\right)}{d t_{j}}
\end{array}\right.
$$

where $t_{j}$ is the center of time period $j, g_{d_{i}}(\cdot)$ models the smooth intraday call arrival pattern that depends on day-of-the-week $d_{i}(=1, \ldots, 5), y_{i}$ is a random effect with an autoregressive structure adjusting for $d_{i}$, and $W_{d_{i}}(t)$ are independent Wiener processes with $W_{d_{i}}(0)=0$ and $\operatorname{var}\left\{W_{d_{i}}(t)\right\}=t$. To fit the model, the authors suggest suitable priors on the parameters, $\alpha_{1}, \ldots, \alpha_{5} ; \tau_{1}^{2}, \ldots, \tau_{5}^{2} ; \sigma^{2} ; \phi^{2}$ and $\beta$, and propose a MCMC algorithm to estimate them.

We also consider the following two models which are related to the above existing approaches. The models may appear to be classical, but they have not been explicitly used in the call center forecasting literature. In addition, we use the two models in the simulation study reported in Section 4.3.

A Multiplicative Model. We assume the following multiplicative (MUL) model,

$$
\left\{\begin{array}{l}
x_{i j}=y_{i} \gamma_{d_{i} j}+\epsilon_{i j}, \quad \sum_{j} \gamma_{d_{i}}=1, \quad \epsilon_{i j} \sim N\left(0, \sigma^{2}\right), \\
y_{i}-\alpha_{d_{i}}=\beta\left(y_{i-1}-\alpha_{d_{i-1}}\right)+\eta_{i}, \quad \eta_{i} \sim N\left(0, \phi^{2}\right), \\
i=1, \ldots, n ; \quad j=1, \ldots, m ; \quad d_{i}=1,2,3,4,5
\end{array}\right.
$$

In the call center context, this model assumes that, on the square-root-transformed scale, the daily total $\left(y_{i}\right)$ follows an $\operatorname{AR}(1)$ model that depends on day-of-the-week $\left(\alpha_{d_{i}}\right)$; each weekday has its own intraday arrival profile $\left(\gamma_{d_{i} j}\right)$. The model is in essence the same as the above Bayesian model used in Weinberg et al. (2007), except that the intraday profiles are not required to be smooth. We provide details about one estimation/forecasting procedure for Model (13) in Appendix A.3. 
An Additive Model. The following additive (ADD) model is assumed on $x_{i j}$,

$$
\left\{\begin{array}{l}
x_{i j}=\mu+\alpha_{i}+\beta_{j}+\gamma_{d_{i} j}+\epsilon_{i j}, \quad \epsilon_{i j} \sim N\left(0, \sigma^{2}\right), \\
\alpha_{i}-a_{d_{i}}=b\left(\alpha_{i-1}-a_{d_{i-1}}\right)+\eta_{i}, \quad \eta_{i} \sim N\left(0, \phi^{2}\right), \\
\sum_{i} \alpha_{i}=\sum_{j} \beta_{j}=\sum_{i} \gamma_{d_{i} j}=\sum_{j} \gamma_{d_{i} j}=\sum_{i j} \gamma_{d_{i} j}=0 \\
i=1, \ldots, n ; \quad j=1, \ldots, m ; \quad d_{i}=1,2,3,4,5
\end{array}\right.
$$

This essentially corresponds to a linear additive model with time-of-day $\left(\beta_{j}\right)$ and daily $\left(\alpha_{i}\right)$

effects as well as their interactions $\left(\gamma_{d_{i} j}\right)$, and the day-of-the-week main effects are assumed to be an $\mathrm{AR}(1)$ process adjusting for day-of-the-week $\left(a_{d_{i}}\right)$. Such a model is also reasonable for our call center application. One estimation/forecasting procedure of Model (14) is detailed in Appendix A.4.

\subsubsection{Dynamic Intraday Updating}

Weinberg et al. (2007) propose an updating algorithm for the Bayesian approach, while the HA/MUL/ADD approaches do not perform any intraday updating. Besides the Bayesian updating, we compare our proposed dynamic updating approach with the following two updating methods.

The Historical Proportion Method. The first method is a simple updating that combines the HA/MUL/ADD forecasts with historical proportions (HP). Suppose the HA forecast for day $n+1$ is $\hat{\mathbf{x}}_{n+1}^{\mathrm{HA}}$. For an updating point $m_{0}$,

- calculate the ratio $R$ between the (square-root-transformed) number of calls received up to time period $m_{0}$ and the number forecasted up to that time,

$$
R=\frac{\sum_{j=1}^{m_{0}} x_{n+1, j}}{\sum_{j=1}^{m_{0}} \hat{x}_{n+1, j}^{\mathrm{HA}}} ;
$$

- update the HA forecasts for the remaining of the day as,

$$
\hat{x}_{n+1, j}^{\mathrm{HP}}=R \hat{x}_{n+1, j}^{\mathrm{HA}}, \quad j=m_{0}+1, \ldots, m .
$$

We can replace the HA forecast with the MUL/ADD forecast in the above algorithm, and refer to the corresponding updates as HPM and HPA, respectively. A non-trivial observation needs to be pointed out here. Due to the specific forms/estimations of the HA model and the MUL model, for the same forecasting day, the MUL forecast turns out to be the HA forecast multiplied by a constant that takes into account the interday correlation. Hence, 
MUL and HA produce the same HP updates, because the constant gets canceled out as one can see from the above algorithm.

The Multiple Regression Method. The second method is based on a multiple linear regression $(\mathrm{MR})$. Let $\mathbf{X}^{e}=\left(\mathbf{x}_{1}^{e}, \ldots, \mathbf{x}_{n}^{e}\right)^{T}$ be a $n \times m_{0}$ matrix containing the historical squareroot-transformed count up to period $m_{0}$, and $\mathbf{X}^{l}=\left(\mathbf{x}_{1}^{l}, \ldots, \mathbf{x}_{n}^{l}\right)^{T}$ be the corresponding $n \times\left(m-m_{0}\right)$ matrix after period $m_{0}$. The MR method works as follows,

- regress $\mathbf{X}^{l}$ on $\mathbf{X}^{e}$ and obtain the regression coefficient matrix as

$$
\left(\mathbf{X}^{e T} \mathbf{X}^{e}\right)^{-1} \mathbf{X}^{e T} \mathbf{X}^{l}
$$

- update the forecast of $\mathbf{x}_{n+1}^{l}$ as the model prediction of $\mathbf{x}_{n+1}^{e}$,

$$
\hat{\mathbf{x}}_{n+1}^{l \mathrm{MR}}=\mathbf{X}^{l T} \mathbf{X}^{e}\left(\mathbf{X}^{e T} \mathbf{X}^{e}\right)^{-1} \mathbf{X}_{n+1}^{e}
$$

Note that here we are updating the multivariate response $\mathbf{x}_{n+1}^{l}$ simultaneously, which is equivalent to updating each element of $\mathbf{x}_{n+1}^{l}$ separately.

\subsection{Two Simulated Examples}

The proposed forecasting approach is data-driven, which assumes no probabilistic model on the underlying data generating process. Here we use two simulation examples to illustrate that our approach is actually very robust to various model assumptions, in the sense that it performs almost as good as the method that uses the true data generating model. On the other hand, the method based on a specific model gives inferior forecast when the model assumed is different from the underlying data generating model. In this case, we refer to the assumed model as a mis-specified model, and the data generating model as the true model. Note that such a distinction of true/mis-specified models is only feasible in simulation studies. For real data, we never know what the true model is.

For the two examples, the multiplicative model (13) and the additive model (14) are respectively considered as the true data generating model. To choose reasonable model parameters, we fit the models to the data described in Section 2 (on the square-root-transformed scale). The obtained parameter estimates are rounded properly for use in the simulations. In particular, some estimated model parameters are reported below,

- for Model (13): $\alpha_{1}=2045, \alpha_{2}=1935, \alpha_{3}=1900, \alpha_{4}=1890, \alpha_{5}=1885, \beta=0.65$, $\sigma=0.5, \phi=35, n=200$ and $m=68$; 
- for Model (14): $\mu=28.5, b=0.65, \sigma=\phi=0.5, n=200$ and $m=68$.

Data sets are generated using each of the two models. Each simulation example is repeated 100 times. During each simulation run, a rolling out-of-sample forecasting exercise is performed with the last 50 days as the forecasting set; and for each day in the forecasting set, the preceding 150 days are used to derive the forecast. Seven forecasting methods are applied to both simulations, including forecasting using the multiplicative model (13), forecasting using the additive model (14), and our methods TS1 to TS5. The parameters involved in all the methods are re-estimated for each update in the rolling forecast exercise for each simulated dataset. Note that, when the data is generated using the multiplicative model, the additive model is a mis-specified model; and vice versa. In both cases, our TS methods do not use the model assumptions.

Figure 2: Comparison of Empirical CDF of Mean RMSE for several forecasting methods under the multiplicative simulation model (13). TS4 and TS5 perform comparably to the true model, while the wrong additive model (14) performs worse.

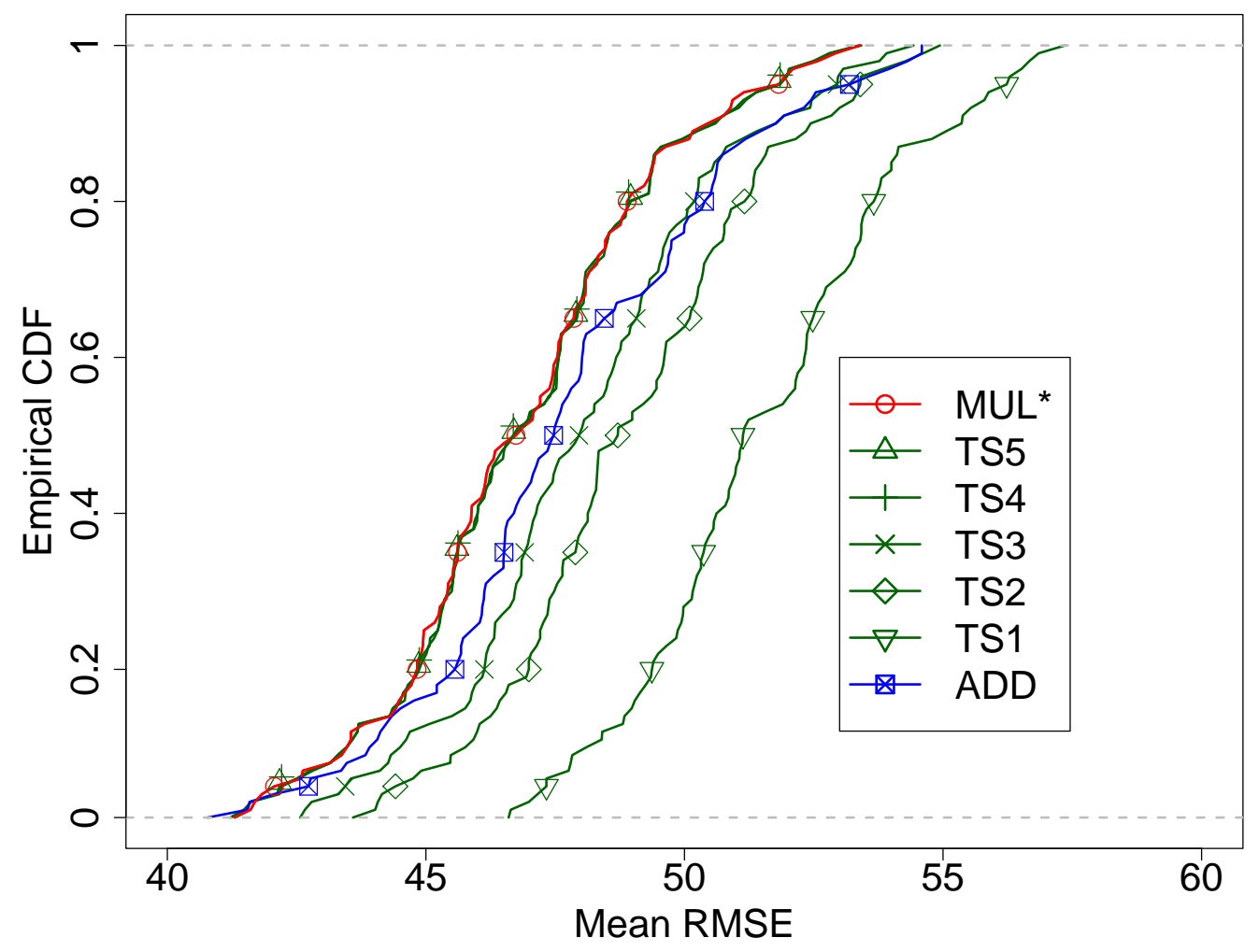

For each simulation run, we calculate the Mean RMSE and Mean MRE (\%) over the forecasting set for each method. Based on the 100 runs, we calculate the empirical cumulative 
Figure 3: Comparison of Empirical CDF of Mean RMSE for several forecasting methods under the additive simulation model (14). TS4 and TS5 perform comparably to the true model, while the wrong multiplicative model (13) performs worse.

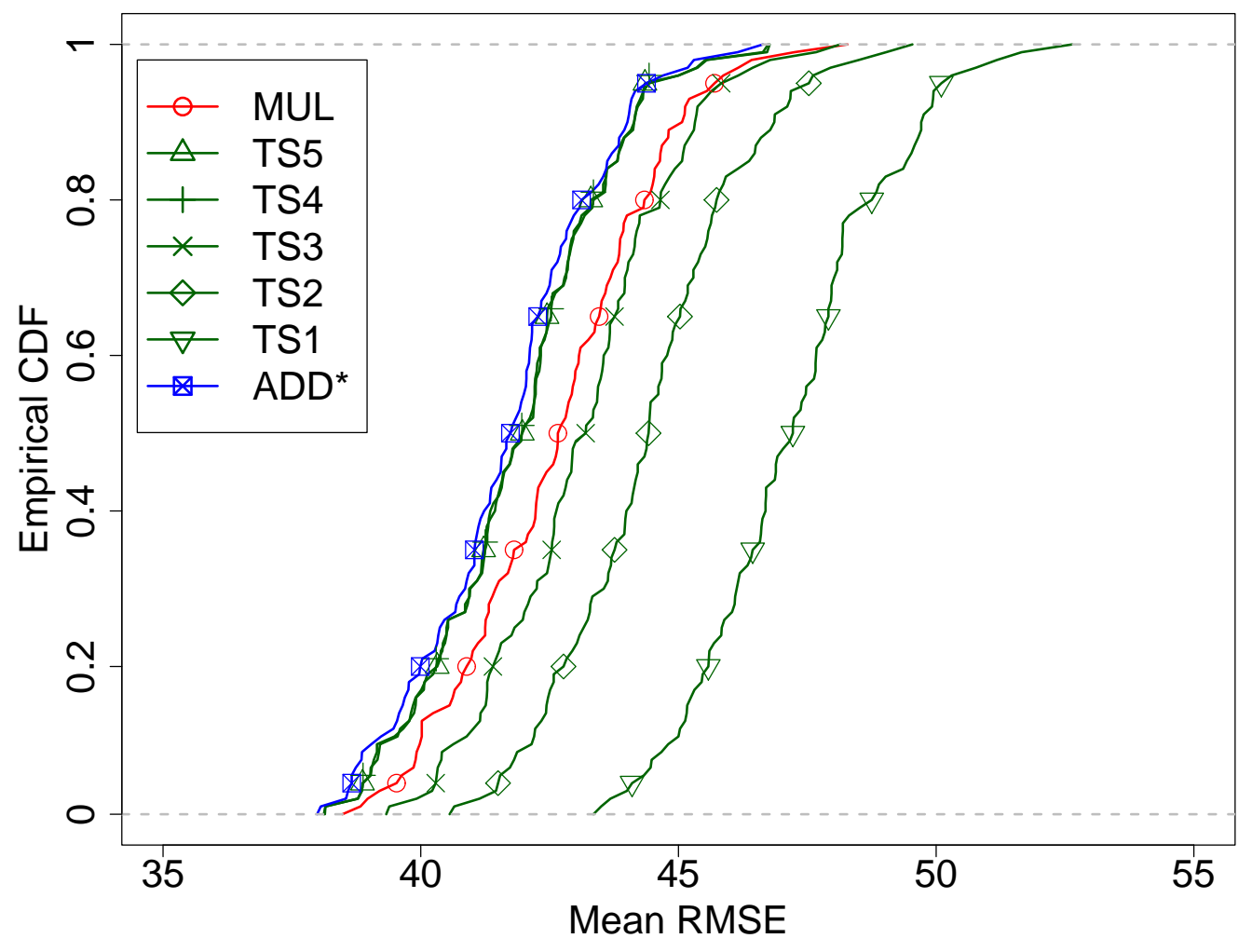

distribution functions (CDF) of the Mean RMSE, which are plotted in Figure 2 when the data are generated from the multiplicative model and in Figure 3 when the data are from the additive model. The true model is highlighted in the legend using an asterisk. The results for Mean MRE (\%) are similar and not shown. To help read these graphs, note that, if the $\mathrm{CDF}$ of one random variable is above that of the other random variable, then the first variable is stochastically smaller than the second one.

We observe that the forecasts based on mis-specified models have stochastically larger Mean RMSE and Mean MRE (\%) than the forecasts based on the correctly specified models. Hence inferior forecasts are obtained when using the mis-specified models. For our proposed TS methods, when the number of feature vectors $K$ increases, both performance measures get stochastically smaller and smaller; and TS4 and TS5 have about the same forecasting performance, which are competitively close to the true model. Note that the CDFs of TS4/TS5 and the true model are hard to distinguish in each plot. The robust good 
performance of the proposed method is important in practice since the proposed method does not require the information of the true underlying model.

\subsection{Case Study I: Our Data}

In this section, we use our data (Section 2) to illustrate the proposed methods for interday forecasting as well as intraday updating. In order to implement the sophisticated Bayesian approach in Weinberg et al. (2007), one needs the expertise with programming MCMC. Hence we did not apply their approach to our data. Instead, we apply our forecasting methods to their data in Section 4.5 to compare with their published results.

As described earlier, there are in total 200 intraday call volume profiles (aggregated into quarter hours) in our data. To perform the rolling-forecast, we use the final 50 days as the forecasting set; for each day in the forecasting set, we use the 150 preceding days as the historical data to re-estimate the model and generate the forecast.

\subsubsection{Interday Forecasting}

Our approach for one-day-ahead forecasting as described in Section 3.2 relies on a dimension reduction by SVD. The original high-dimensional vector time series is represented using a small number $K$ of basis vectors. In this study, we consider $K=1, \ldots, 4$ and denote the methods as TS1, ..., TS4 respectively. The scree plot based on the first 150 days suggests that we only need to consider up to five pairs of feature vectors, which makes sense in that the five weekdays appear to have different arrival patterns (Figure 1(a)). We note that TS4 and TS5 turn out to have very similar forecasting performance.

To help interpret the feature factors, Figure 4 plots the first four pairs of intraday feature vectors and interday feature series, all of which are extracted from the first 150 days. As it turns out, the first intraday feature summarizes the average intraday call arrival pattern during a regular weekday; while the first interday feature illustrates a strong weekly effect, and is actually highly correlated with total daily call volume. The remaining pairs are second-order effects as indicated by the much smaller scales of the second to fourth interday feature series. The corresponding intraday features capture certain contrasts between arrival patterns of different within-day segments. For example, the second intraday feature compares morning arrivals with afternoon and evening arrivals; when combined with the second interday feature, they suggest the following specific phenomenon: Fridays usually have above average 
call volumes in early mornings and below average volumes in late afternoons and evenings, while the opposite is true for Mondays.

Further analysis of the interday feature series suggest that they can be modeled separately by an $\mathrm{AR}(1)$ time series model with a day-of-the-week effect. Specifically, for $k=1, \ldots, 4$, we model the series $\left\{\beta_{i k}\right\}$ by

$$
\beta_{i k}=a_{k}\left(d_{i-1}\right)+b_{k} \beta_{i-1, k}+\epsilon_{i}
$$

where the varying intercept $a_{k}\left(d_{i-1}\right)$ depends on the day-of-the-week of day $i-1$, and the $\epsilon_{i}$ 's are independent identically distributed errors with mean 0 . The same model has been obtained in Shen and Huang (2005) when analyzing the arrival data from the same call center for a different year. A closely related model is used in Weinberg et al. (2007).

Table 1 compares summary statistics of the RMSE and MRE of the forecasts from the HA/MUL/ADD methods and the four TS methods. The best forecasting method is highlighted with boldface for each summary statistic separately. Based on RMSE, all TS methods show substantial improvement over the benchmark HA, while TS4 gives the best overall performance. As for MRE, TS1 performs comparable with HA, because MRE is more sensitive to forecasting performance at the beginning and the end of the day where call volumes are low. At least two feature vectors are needed to capture the difference among different weekdays in those regions. Both ADD and MUL also improve significantly over HA, with ADD performing similar to TS2 and MUL similar to TS4.

Table 1: Summary statistics (mean, median, lower quartile Q1, upper quartile Q3) of RMSE and MRE in a rolling forecast exercise. The forecasting set contains 50 days.

\begin{tabular}{|c|c|c|c|c|c|c|c|c|}
\hline & \multicolumn{4}{|c|}{$\overline{\overline{\text { RMSE }}}$} & \multicolumn{4}{|c|}{ "MRE (\%) } \\
\hline & Q1 & Median & Mean & Q3 & Q1 & Median & Mean & Q3 \\
\hline HA & 43.81 & 65.57 & 72.89 & 90.04 & 5.2 & 6.7 & 7.3 & 8.7 \\
\hline ADD & 41.71 & 52.56 & 59.28 & 63.67 & 4.8 & 5.6 & 6.5 & 6.9 \\
\hline MUL & 41.55 & 48.54 & 57.49 & 59.33 & 4.6 & 5.3 & 6.1 & 6.6 \\
\hline TS1 & 42.91 & 53.52 & 60.09 & 63.76 & 5.2 & 6.6 & 7.4 & 8.6 \\
\hline TS2 & 39.57 & 51.29 & 57.96 & 61.04 & 4.9 & 5.6 & 6.6 & 7.1 \\
\hline TS3 & 42.90 & 51.96 & 57.65 & 58.01 & 4.8 & 5.5 & 6.4 & 6.9 \\
\hline TS4 & 41.85 & 48.23 & 56.88 & 59.27 & 4.6 & 5.3 & 6.2 & 6.7 \\
\hline
\end{tabular}

We also compare the performance of the $95 \%$ bootstrap prediction intervals for our TS methods. The bootstrap procedure described in Section 3.2 is implemented with $B=1000$. 
Figure 4: The first four pairs of intraday feature vectors and interday feature series.
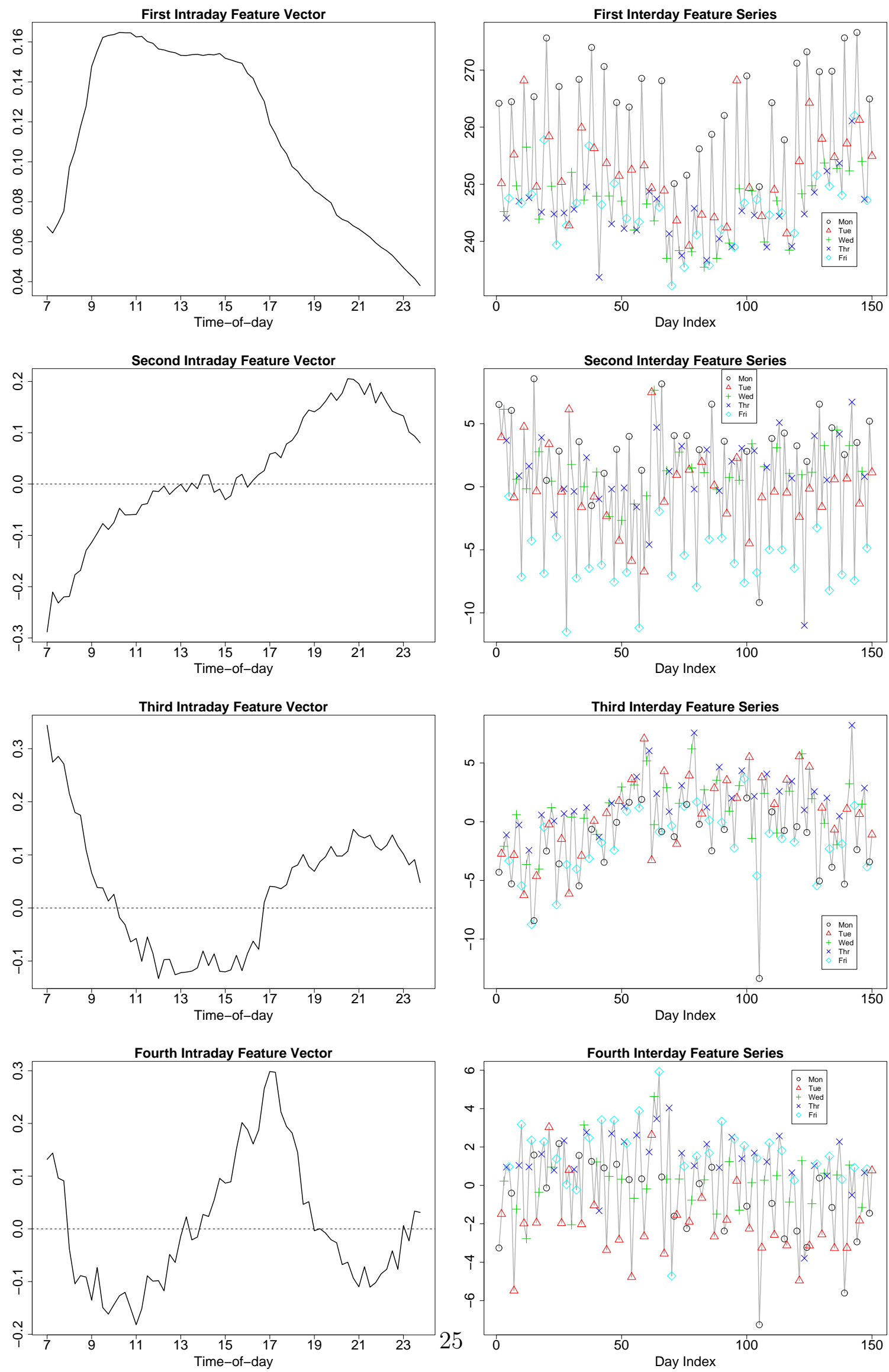
The average empirical coverage probabilities of the prediction intervals turn out to be $94.4 \%$, 94.9\%, 94.8\% and 94.6\% respectively, which are quite accurate. Table 2 compares the distribution of the average prediction interval width among the four TS approaches, which suggests that the intervals get stochastically narrower as one increases the number of components, $K$, in the forecasting model; hence the forecasting becomes more and more precise.

Table 2: Summary statistics (mean, median, lower quartile Q1, upper quartile Q3) of Average Width in a rolling forecast exercise. The forecasting set contains 50 days. TS4 performs the best. The intervals are stochastically narrower as one increases $K$.

\begin{tabular}{ccccc}
\hline \hline & Q1 & Median & Mean & Q3 \\
\hline TS1 & 234.2 & 242.0 & 243.1 & 249.4 \\
TS2 & 225.4 & 232.3 & 233.3 & 242.4 \\
TS3 & 220.3 & 226.9 & 227.8 & 234.0 \\
TS4 & $\mathbf{2 1 5 . 1}$ & $\mathbf{2 2 1 . 8}$ & $\mathbf{2 2 3 . 1}$ & $\mathbf{2 2 9 . 7}$ \\
\hline
\end{tabular}

\subsubsection{Dynamic Intraday Updating}

For each day in the forecasting set, we consider dynamically updating the forecast for the rest of the current day at every half hour starting from 8:00AM, using available information up to that time. In addition to the HP/HPM/HPA and MR updating methods (Section 4.2.2), we consider the three updating methods described in Section 3.3, namely TS, LS, and PLS; see (5), (7) and (11). We expect that the PLS method should give the best forecast performance. This is confirmed empirically below. To implement these three methods, we need to choose $K$, the number of intraday feature vectors for dimension reduction. In light of the one-dayahead forecasting performance presented above in Section 4.4.1, we decide to use $K=4$ and the three methods are therefore denoted as TS4, LS4, and PLS4. For each day in the forecasting set, we use its 150 preceding days to derive the time series forecast TS4. We employ the current day's incoming call volume information in addition to the 150 preceding days to produce the LS4 and PLS4 forecasts. The intraday updating starts at 8:00AM, corresponding to $m_{0}=4$, in order to have enough data to estimate the parameters for LS.

To use the PLS updating approach, we need to decide on the value of the penalty parameter $\lambda$ at each updating point. To this end, we use the beginning 150 days in our data set as the training set, and perform an out-of-sample rolling forecast exercise on this training set. Note that this training set does not overlap with the forecasting set we initially hold 
out for out-of-sample forecast evaluation. For the purpose of selecting $\lambda$, the last one third (i.e., 50 days) of the training set is used as a rolling hold-out sample. For a given day in this hold-out sample, we use the preceding 100 days to extract the interday feature series to generate the time series forecast TS4. The extracted intraday feature vectors and the obtained TS4 forecast are used for all updating points within that day.

Fix an updating point, for example, 10:00AM. For each given $\lambda$, we construct the PLS updating for the given day. Pick a performance measure such as RMSE or MRE. Compute the performance measure for all days in the hold-out sample and calculate the average value. Select $\lambda$ by minimizing this average performance measure over a grid of candidate values. In this study, we choose $\lambda$ from $\left\{0,10, \ldots, 10^{9}\right\}$. When $\lambda=0$, the PLS approach is the same as the LS approach. As $\lambda$ goes to infinity, the PLS approach should converge to the TS approach, which is essentially achieved when $\lambda=10^{9}$ in our study. Second-round tuning can be carried out over a finer grid around the selected $\lambda$ to improve performance if needed.

For a given updating point, the selected $\lambda$ is kept fixed for all days in the forecasting set to generate the PLS forecast for the corresponding updating. We observe that, for every selection criterion (RMSE or MRE), the selected $\lambda$ in general has a decreasing trend as the updating time progresses into the later part of the day. Note that a smaller $\lambda$ value would let the PLS forecast put more weight on the LS forecast. Thus the observed decreasing trend for the selected $\lambda$ makes intuitive sense. Because, as time progresses, more information is available about the current day's call arrival pattern, and the forecaster needs less influence from the TS forecast.

Figure 5 plots the average of RMSE as functions of the updating points for the seven approaches, where the averages are taken over the 50 days in the forecasting set. TS4 is treated as the benchmark as it essentially performs no intraday updating. It just uses the information up to the end of the previous day and does not employ the current day's information. Note that RMSE is calculated at each updating point since we are interested in the performance of the updated forecasts at all time periods after the updating point. For PLS4, RMSE is used to select $\lambda$ as it is the performance measure in Figure 5.

From the plot, PLS4 is clearly the winner, which has the smallest error measure at all updating points. The improvement over TS4 starts to become really significant as early as updating at 10:00AM, which is 3 hours into the working day. Thus, the significant improvement of PLS4 over TS4 shows the benefit of dynamic updating using the current day's information. One can also see that LS4 performs rather badly at the beginning of the 
Figure 5: Comparison of Mean RMSE as functions of the updating points, which are calculated over the 50 days in the forecasting set. PLS4 performs the best.

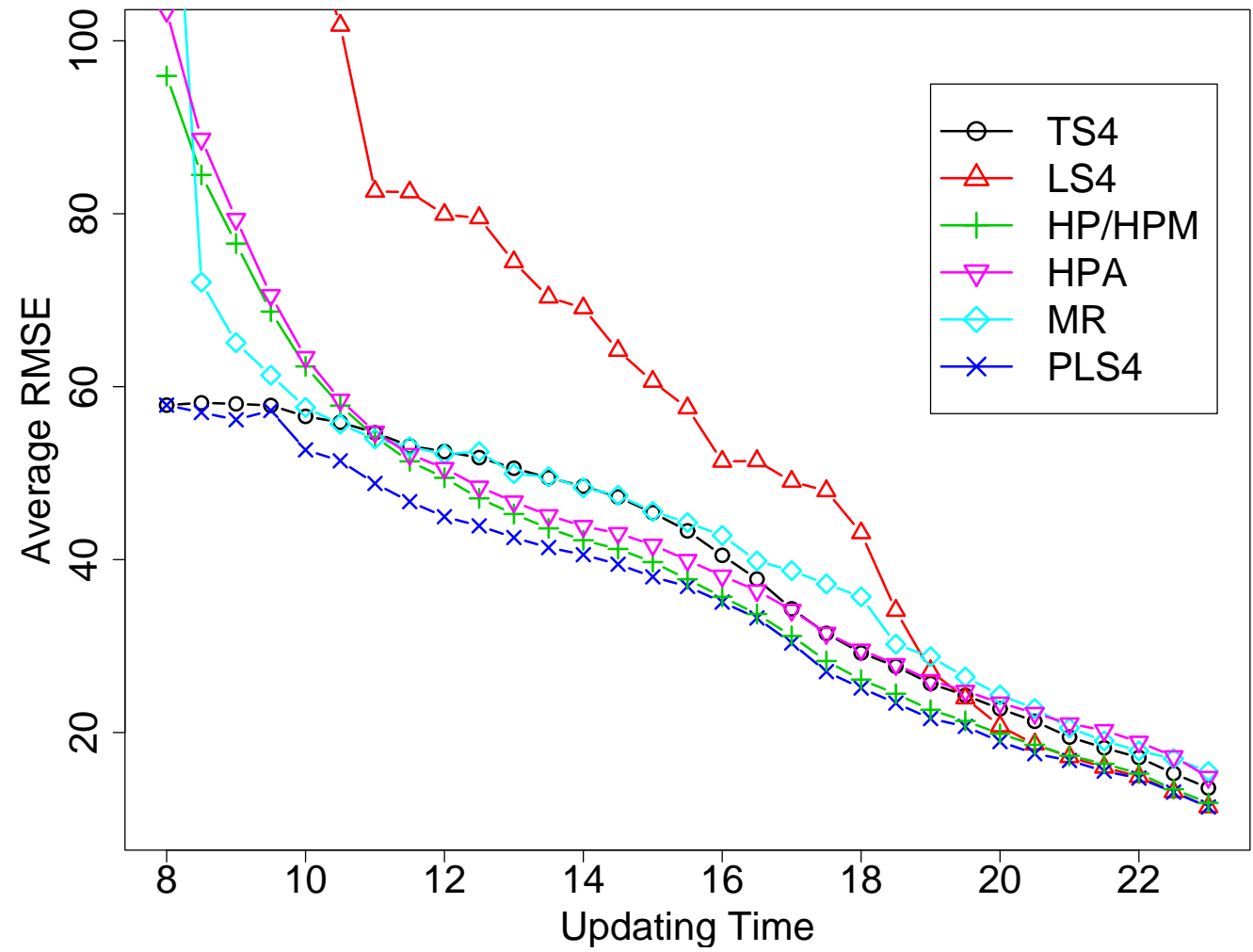

day, which is due to the high collinearity between the initial parts of the higher order intraday feature vectors. This also justifies the necessity for penalization. The MR updating performs no better than TS4, and much worse prior to 10:00AM; this suggests that the interday dependence plays a much bigger role in improving forecasting, which isn't incorporated in MR. As discussed in Section 4.2.2, HP and HPM lead to the same updates. The HP/HPM approach behaves rather badly in early mornings, and starts to beat TS4 at 11:00AM; it performs worse than PLS4 until 16:00PM, when the performance converges to PLS4's. It seems that a call center manager needs enough information for the present day in order for the HP/HPM updating to work well. To the extent that she has that information, HP/HPM is a nice candidate to perform updating due to its simplicity. The HPA approach performs uniformly worse than HP/HPM.

Below, we look at the 10:00AM updating and the 12:00PM updating, in order to get some idea about how different approaches perform for individual updating. Table 3 presents summary statistics of the RMSE of the forecasts from TS4, LS4, HP/HPM, HPA, MR 
and PLS4. The averages are calculated over the 50 days in the forecasting set. For a fair comparison, we only use data after 12:00PM when calculating the RMSE. The superior performance of PLS4 over the other methods is again quite clear. The LS4 method has the worst performance, even much worse than TS4. For every method except TS4, updating later always improves the forecasting accuracy. Note that TS4 performs no intraday updating.

Table 3: Summary statistics (mean, median, lower quartile Q1 and upper quartile Q3) of RMSE for the 10:00AM and 12:00PM updatings. PLS4 outperforms the other methods.

\begin{tabular}{|c|c|c|c|c|c|c|c|c|}
\hline & \multicolumn{4}{|c|}{ 10:00AM updating } & \multicolumn{4}{|c|}{ 12:00PM updating } \\
\hline & Q1 & Median & Mean & Q3 & Q1 & Median & Mean & Q3 \\
\hline TS4 & 36.15 & 41.74 & 52.55 & 54.60 & 36.15 & 41.74 & 52.55 & 54.60 \\
\hline LS4 & 83.28 & 106.90 & 119.20 & 128.10 & 54.92 & 73.83 & 79.90 & 97.46 \\
\hline $\mathrm{HP} / \mathrm{HPM}$ & 37.64 & 49.76 & 59.30 & 70.10 & 35.68 & 39.60 & 49.46 & 49.74 \\
\hline HPA & 37.66 & 52.17 & 60.13 & 71.11 & 35.84 & 41.05 & 50.57 & 50.38 \\
\hline MR & 41.06 & 48.24 & 56.50 & 62.96 & 40.45 & 47.04 & 52.21 & 52.20 \\
\hline PLS4 & 37.04 & 42.08 & 50.77 & 58.07 & 33.69 & 37.87 & 44.96 & 44.25 \\
\hline
\end{tabular}

Intraday updating not only can reduce forecasting errors, but also narrow the width of forecast intervals while maintaining the same coverage probability. To illustrate this point, Table 4 reports a width comparison of $95 \%$ forecast intervals among three forecasting approaches: TS4 where no updating is performed, PLS4 with 10:00AM updating, and PLS4 with 12:00PM updating. The intervals are stochastically narrower as more observations are used for the updating. The two updatings reduce the average interval width by $11.5 \%$ and $9.0 \%$ sequentially. The average empirical coverage probability of the three intervals are respectively $93.7 \%, 93.3 \%$ and $94.0 \%$, close to the nominal value.

Table 4: Summary statistics (mean, median, lower quartile Q1 and upper quartile Q3) of Average Width of $95 \%$ forecast intervals among three approaches: TS4 (i.e. no updating), PLS4 (10:00AM updating) and PLS4 (12:00PM updating).

\begin{tabular}{ccccc}
\hline \hline & Q1 & Median & Mean & Q3 \\
\hline TS4 & 193.1 & 199.8 & 200.1 & 205.2 \\
PLS4 (10:00AM) & 171.2 & 175.8 & 177.1 & 182.0 \\
PLS4 (12:00PM) & $\mathbf{1 5 5 . 5}$ & $\mathbf{1 6 1 . 2}$ & $\mathbf{1 6 1 . 2}$ & $\mathbf{1 6 4 . 6}$ \\
\hline
\end{tabular}

We recall that the PLS criterion (8) involves two terms. To understand their relative importance, one can evaluate the criterion at the selected $\lambda$ and $\hat{\boldsymbol{\beta}}_{n+1}^{\mathrm{PLS}}$, and calculate the 
percentage of each term relative to the total. For the current study, the first term accounts for $89.2 \%$ and $90.1 \%$ of the total for the 10:00AM and 12:00PM updatings, respectively. The first term becomes more important as one postpones the updating, which makes sense.

\subsection{Case Study II: Weinberg's Data}

In this section, we compare our method with the Bayesian Gaussian (BG) approach proposed by Weinberg et al. (2007). To compare with their published results, we apply our method to the same cleaned data used by Weinberg et al. Their data consist of 5-minute aggregated call volume profiles from 7:00AM to 9:05PM for 164 days; thus the dimensionality is 169 . In Weinberg et al. (2007), the data set was cleaned by excluding anomalous days such as holidays. For the rolling-forecast, the final 64 days are used as the forecasting set. For each day in the forecasting set, its 100 preceding days are considered as the historical data.

\subsubsection{Interday Forecasting}

For our forecasting approach, we consider two methods, one using three intraday feature vectors (TS3) and the other using five (TS5). The reason of such a choice is that the BG approach assumes a separate intraday pattern for each weekday, which corresponds to the model with five intraday feature vectors, and the use of three intraday feature vectors has already given good forecast results. Note that Weinberg et al. constrain the intraday patterns to be smooth while we do not impose such a constraint. We again use the varying-coefficient $\mathrm{AR}(1)$ model to forecast the interday feature series.

Table 5 reports summaries of the RMSE and MRE of the forecasts from various competing approaches. The results for BG are quoted from Weinberg et al. (2007). As expected, all the approaches improve greatly over HA. It is also observed that our approaches perform very competitively with the BG approach. Through personal communication, we find out that the Bayesian approach might take a long time to run due to the many MCMC iterations needed. As a comparison, our approach is computationally fast, which makes it amenable for realtime updating. The results also suggest that a forecasting model with three intraday features (TS3) might be sufficient. Both ADD and MUL methods perform similar to our TS3 approach with TS3 using the least number of intraday features. We point out in Section 4.2.1 that the only major difference between MUL and BG is that BG assumes smooth intraday patterns. The close performance between them suggests that smoothing is not necessary for the one-day-ahead forecasting of the current data. 
Table 5: Summary statistics (mean, median, lower quartile Q1, upper quartile Q3) of RMSE and MRE for six competing approaches: HA, ADD, MUL, BG, TS3 and TS5. Note that the forecasting exercise is performed on the data used in Weinberg et al. (2007).

\begin{tabular}{|c|c|c|c|c|c|c|c|c|}
\hline & \multicolumn{4}{|c|}{$\overline{\text { RMSE }}$} & \multicolumn{4}{|c|}{ MRE (\%) } \\
\hline & Q1 & Median & Mean & Q3 & Q1 & Median & Mean & Q3 \\
\hline$\overline{\mathrm{HA}}$ & 16.22 & 19.12 & 21.32 & 21.82 & 7.8 & 9.3 & 10.1 & 11.6 \\
\hline ADD & 14.49 & 15.89 & 18.46 & 19.84 & 7.0 & 7.6 & 8.5 & 8.7 \\
\hline MUL & 14.42 & 15.81 & 18.28 & 19.97 & 6.9 & 7.3 & 8.4 & 8.7 \\
\hline BG & 14.25 & 15.83 & 18.28 & 19.83 & 7.0 & 7.4 & 8.4 & 8.5 \\
\hline TS3 & 14.34 & 15.76 & 18.19 & 20.22 & 6.9 & 7.5 & 8.5 & 8.8 \\
\hline TS5 & 14.20 & 15.82 & 18.16 & 19.99 & 6.8 & 7.3 & 8.3 & 8.6 \\
\hline
\end{tabular}

\subsubsection{Dynamic Intraday Updating}

Weinberg et al. (2007) also propose a Bayesian procedure to perform intraday updating. In this section, we are interested in comparing their updating procedure (BG) with our penalized least squares updating approach (PLS3).

The penalty parameter $\lambda$ for the PLS updating is chosen using the data-driven procedure described in Section 4.4.2. We use the last 30 days of the 100-day training set as a rolling hold-out sample. For each day in this sample, its preceding 70 days and early part of the current day are used to generate the updated forecast for some candidate values of $\lambda$. We then select $\lambda$ as the value that minimizes some performance measure averaged over the hold-out sample.

To compare with the results reported in their paper, we look at the 10:00AM updating and the 12:00PM updating, and the forecasting measures are calculated only using data after 12:00PM. Table 6 reports the summaries of RMSE for the BG approach (quoted from their paper) and PLS3, which suggest that PLS3 improves slightly over BG in all cases.

The Bayesian approach naturally generates prediction intervals using the MCMC sample. To compare the performance of the intervals, summaries of the Average Width for 95\% prediction intervals are presented in Table 6 for BG and PLS3. We observe that PLS3 with 12:00PM updating leads to the narrowest intervals in all categories. All the methods have approximately the right coverage probability with the empirical averages around $94 \%$. 
Table 6: Summary statistics (mean, median, lower quartile Q1, upper quartile Q3) of RMSE and Average Width of $95 \%$ prediction intervals for BG and PLS3. Note that the forecasting exercise is performed on the data used in Weinberg et al. (2007).

\begin{tabular}{|c|c|c|c|c|c|c|c|c|c|}
\hline & \multicolumn{4}{|c|}{$\overline{\text { RMSE }}$} & \multicolumn{4}{|c|}{ 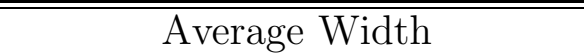 } \\
\hline & & $\overline{\mathrm{Q} 1}$ & Median & Mean & $\overline{\mathrm{Q} 3}$ & $\overline{\mathrm{Q} 1}$ & Median & Mean & $\overline{\mathrm{Q} 3}$ \\
\hline \multirow{2}{*}{ 10:00AM } & $\overline{\mathrm{BG}}$ & 14.00 & 15.50 & 17.86 & 19.87 & 58.87 & 60.74 & 61.11 & 63.50 \\
\hline & PLS3 & 13.31 & 14.87 & 16.48 & 17.25 & 59.58 & 61.17 & 61.32 & 62.81 \\
\hline \multirow{2}{*}{ 12:00PM } & $\overline{B G}$ & 13.56 & 14.80 & 16.59 & 16.58 & 58.78 & 60.42 & 60.80 & 62.34 \\
\hline & PLS3 & 13.33 & 14.60 & 16.13 & 16.39 & 58.14 & 59.27 & 59.56 & 61.38 \\
\hline
\end{tabular}

\section{Conclusion}

Our approach to forecasting call arrivals is based on the viewpoint that the intraday call volume profiles form a vector time series. This point of view represents clearly that the data have a two-way temporal structure, that is, the interday and intraday variations. Such a structure exists in other applications as well. The SVD, used as a dimensionality reduction tool, effectively separates out these two types of variations. The intraday variation is summarized by a few intraday feature vectors that can be extracted from historical data, while the interday variation is modeled by a few interday feature time series (see Section 3.1). The effectiveness of SVD for dimension reduction is shown in our data examples: three pairs of interday and intraday features are sufficient to generate good forecasts for a 68 (or 169)-dimensional time series.

Assuming that the intraday features won't change in the near future, the problem of forecasting intraday profiles reduces to the problem of forecasting the interday features. We propose to use univariate time series techniques to forecast the interday feature series in order to generate one- or multi-day-ahead forecasts. For dynamic intraday updating, we have considered three ways to update the interday feature forecasts: 1 . Apply the interday forecast, using information available up to the end of the previous day (i.e. no updating); 2. Fit a least squares regression using the observed portion of the current day's volume profile on the corresponding components of the intraday feature vectors (i.e. LS updating); 3. Combine the current day's available information with the previous day's information using penalized least squares (i.e. PLS updating). Not surprisingly, the third method (PLS) performs the best and is the recommended method.

Our methods show improvements over various alternatives in two out-of-sample fore- 
casting comparisons using real data. Despite the good performance, our methods are not much more complicated than the alternatives. They are easy to implement. SVD can be obtained using any software that does matrix computation such as MATLAB and SPLUS/R; and solving penalized least squares is as straightforward as performing a ridge regression. The $\mathrm{R}$ codes for performing our forecasting and updating methods, as well as the data, are available from the authors upon request. Our methods generate very competitive or slightly better forecasts when compared with a sophisticated Bayesian approach recently proposed by Weinberg et al. (2007). Another advantage of our approach is its fast computation, which makes it a feasible technique for real-time forecasting and updating.

A natural direction for future research is to combine our dimensionality reduction idea with stochastic modeling, such as modeling the arrival process as an inhomogeneous Poisson process (Brown et al. 2005). In this paper, all the methods considered employ a square-root transformation to overcome the non-constant variance problem. The data transformation is not necessary if a distribution based approach is employed in the spirit of stochastic modeling. One possibility is to model the number of arrivals as a Poisson random variable with a random rate possessing both interday and intraday dependence, which we can model using the dimension reduction idea in this paper. Since the Poisson rate is not observable, such an approach will be inevitably more complex than the current approach. It would be interesting to investigate whether the distributional approach can yield better forecasts.

The current paper focuses on one-day-ahead forecasting and intraday dynamic updating. One closely-related interesting problem is two-week-ahead forecasting, which has gained some research interests lately (Taylor 2007, Aldor-Noiman 2006). However, existing empirical results remain rare, and a careful systematic study is needed. We are currently working on extending our framework to this problem, and initial empirical analysis has shown promising results.

\section{Appendix}

\section{A.1 Bootstrapping the $\beta$ 's in (4) of Section 3.2.2}

For each $k$, we describe how to obtain the $B$ bootstrap series $\left\{\hat{\beta}_{n+1, k}^{b}, \ldots, \hat{\beta}_{n+h, k}^{b}\right\}, 1 \leq b \leq B$, as used in (4). Suppose the time series model for $\left\{\beta_{i k}\right\}$ can be written as

$$
\beta_{i k}=\mathcal{M}\left(\mathcal{H}_{i k}\right)+\epsilon_{i k}
$$


where $\mathcal{H}_{i k}=\left\{\beta_{i-1, k}, \beta_{i-2, k}, \ldots, \beta_{1, k}\right\}$ represents the collection of historical data from the series $\left\{\beta_{i k}\right\}$ up to time $i-1, \mathcal{M}$ represents the function that gives the prediction of $\beta_{i k}$ based on the historical data, and $\epsilon_{i k}$ is the model error. For example, for an AR(1) model,

$$
\mathcal{M}\left(\mathcal{H}_{i k}\right)=a_{0}+a_{1} \beta_{i-1, k}
$$

Let $\widehat{\mathcal{M}}$ denote the fitted function using the data and let $\hat{\epsilon}_{i k}=\beta_{i k}-\widehat{\mathcal{M}}\left(\mathcal{H}_{i k}\right)$ be the fitted model errors. The bootstrapped model errors $\epsilon_{n+1, k}^{b}, 1 \leq b \leq B$, are obtained by sampling with replacement from the set $\left\{\hat{\epsilon}_{n, k}, \hat{\epsilon}_{n-1, k}, \ldots, \hat{\epsilon}_{1, k}\right\}$. A bootstrap sample of $\beta_{n+1, k}$ is then obtained using $\beta_{n+1, k}^{b}=\widehat{\mathcal{M}}\left(\mathcal{H}_{n+1, k}\right)+\epsilon_{n+1, k}^{b}, 1 \leq b \leq B$.

We generate the bootstrap sample of $\beta_{n+h, k}$ for $h>1$ in a sequential manner, assuming availability of $\left\{\beta_{n+1, k}^{b}, \ldots, \beta_{n+h-1, k}^{b}\right\}$. The historical information set used to generate $\beta_{n+h, k}$ depends on $b$ and is defined as $\mathcal{H}_{n+h, k}^{b}=\left\{\beta_{n+1, k}^{b}, \ldots, \beta_{n+h-1, k}^{b}\right\} \cup \mathcal{H}_{n+1, k}$. The bootstrap model errors $\epsilon_{n+h, k}^{b}, 1 \leq b \leq B$, are obtained by sampling with replacement from the set $\left\{\hat{\epsilon}_{n, k}, \hat{\epsilon}_{n-1, k}, \ldots, \hat{\epsilon}_{1, k}\right\}$. A bootstrap sample of $\beta_{n+h, k}$ is then generated using $\beta_{n+h, k}^{b}=$ $\widehat{\mathcal{M}}\left(\mathcal{H}_{n+h, k}\right)+\epsilon_{n+h, k}^{b}, 1 \leq b \leq B$.

\section{A.2 Proof of Theorem 1}

This can be easily seen as follows,

$$
\begin{aligned}
\hat{\boldsymbol{\beta}}_{n+1}^{\mathrm{PLS}} & =\left(\mathbf{F}^{e T} \mathbf{F}^{e}+\lambda \mathbf{I}\right)^{-1} \mathbf{F}^{e T} \mathbf{F}^{e}\left(\mathbf{F}^{e T} \mathbf{F}^{e}\right)^{-1} \mathbf{F}^{e T} \mathbf{x}_{n+1}^{e}+\lambda\left(\mathbf{F}^{e T} \mathbf{F}^{e}+\lambda \mathbf{I}\right)^{-1} \hat{\boldsymbol{\beta}}_{n+1}^{\mathrm{TS}} \\
& =\left(\mathbf{F}^{e T} \mathbf{F}^{e}+\lambda \mathbf{I}\right)^{-1} \mathbf{F}^{e T} \mathbf{F}^{e} \hat{\boldsymbol{\beta}}_{n+1}^{\mathrm{LS}}+\lambda\left(\mathbf{F}^{e T} \mathbf{F}^{e}+\lambda \mathbf{I}\right)^{-1} \hat{\boldsymbol{\beta}}_{n+1}^{\mathrm{TS}} \\
& \equiv A(\lambda) \hat{\boldsymbol{\beta}}_{n+1}^{\mathrm{LS}}+(I-A(\lambda)) \hat{\boldsymbol{\beta}}_{n+1}^{\mathrm{TS}} . \square
\end{aligned}
$$

\section{A.3 Estimation and Forecasting of Model (13)}

The model is multiplicative, which means that one need to use nonlinear least squares to derive estimates. Below we provide a set of simple estimates, which are close to the true least squares estimates (Brown et al. 2005),

$$
\left\{\begin{array}{l}
\hat{y}_{i}=x_{i}=\sum_{j} x_{i j} ; \quad \hat{\alpha}_{d_{i}}=\frac{\sum_{i^{\prime}: d_{i^{\prime}}=d_{i}} x_{i}}{\#\left\{i^{\prime}: d_{i^{\prime}}=d_{i}\right\}} ; \\
\hat{\gamma}_{d_{i j} j}=\frac{\sum_{i^{\prime}: d_{i^{\prime}}=d_{i}} x_{i^{\prime} j}}{\#\left\{i^{\prime}: d_{i^{\prime}}=d_{i}\right\} \hat{\alpha}_{d_{i}}} ; \\
\text { The } \operatorname{AR}(1) \text { coefficient } \beta \text { can then be estimated using linear regression. }
\end{array}\right.
$$

For day $n+1$, the forecast is then

$$
x_{n+1, j}=\hat{y}_{n+1} \hat{\gamma}_{d_{n+1} j},
$$

where $\hat{y}_{n+1}$ is forecasted to be $\hat{\alpha}_{d_{n+1}}+\hat{\beta}\left(\hat{y}_{n}-\hat{\alpha}_{d_{n}}\right)$. 


\section{A.4 Estimation and Forecasting of Model (14)}

Here we provide a set of least squares estimates for the model parameters:

$$
\left\{\begin{array}{l}
\hat{\mu}=\bar{x}_{. .}=\frac{\sum_{i j} x_{i j}}{n m} ; \\
\hat{\alpha}_{i}=\bar{x}_{i .}-\bar{x}_{. .}=\frac{\sum_{j} x_{i j}}{m}-\bar{x}_{. .} ; \quad \hat{\beta}_{j}=\bar{x}_{. j}-\bar{x}_{. .}=\frac{\sum_{i} x_{i j}}{n}-\bar{x}_{. .} ; \\
\hat{a}_{d_{i}}=\frac{\sum_{i^{\prime}: d_{i^{\prime}}=d_{i}} \bar{x}_{i^{\prime}}}{\#\left\{i^{\prime}: d_{i^{\prime}}=d_{i}\right\}}-\bar{x}_{. .} ; \\
\hat{\gamma}_{d_{i j} j}=\frac{\sum_{i^{\prime}: d_{i^{\prime}}=d_{i}} \bar{x}_{i^{\prime} j}}{\#\left\{i^{\prime}: d_{i^{\prime}}=d_{i}\right\}}-\hat{\mu}-\hat{a}_{d_{i}}-\hat{\beta}_{j} ; \\
\text { The } \operatorname{AR}(1) \text { coefficient } b \text { can then be estimated using linear regression. }
\end{array}\right.
$$

Due to the normality assumption of the model errors, the above estimates should be close to the maximum likelihood estimates.

For day $n+1$, the forecast is then

$$
x_{n+1, j}=\hat{\mu}+\hat{\alpha}_{n+1}+\hat{\beta}_{j}+\hat{\gamma}_{d_{n+1} j},
$$

where $\hat{\alpha}_{n+1}$ is forecasted to be $\hat{a}_{d_{n+1}}+\hat{b}\left(\hat{\alpha}_{n}-\hat{a}_{d_{n}}\right)$.

\section{Acknowledgments}

The authors want to extend grateful thanks to the Editor, the Associate Editor and three reviewers whose comments have greatly improved the scope and presentation of the paper. Thanks are also due to Larry Brown, Noah Gans, Avi Mandelbaum and Linda Zhao for their valuable suggestions. Haipeng Shen's work is partially supported by National Science Foundation (NSF) grant DMS-0606577. Jianhua Z. Huang's work is partially supported by NSF grant DMS-0606580.

\section{References}

Aldor-Noiman, S. 2006. Forecasting demand for a telephone call center: Analysis of desired versus attainable precision (master's thesis).

Andrews, B. H., S. M. Cunningham. 1995. LL Bean improves call-center forecasting. Interfaces 25 $1-13$.

Avramidis, A. N., A. Deslauriers, P. L'Ecuyer. 2004. Modeling daily arrivals to a telephone call center. Management Science 50 896-908.

Betts, A., M. Meadows, P. Walley. 2000. Call centre capacity management. International Journal of Service Industry Management 11 185-196.

Bianchi, L., J. Jarrett, R. C. Hanumara. 1993. Forecasting incoming calls to telemarketing centers. Journal of Business Forecasting Methods and Systems 12 3-12. 
Bianchi, L., J. Jarrett, R. C. Hanumara. 1998. Improving forecasting for telemarketing centers by ARIMA modelling with intervention. International Journal of Forecasting 14 497-504.

Box, G. E. P., G. M. Jenkins, G. C. Reinsel. 1994. Time Series Analysis: Forecasting and Control. Third Edition. Prentice Hall, New Jersey.

Brown, L. D., N. Gans, A. Mandelbaum, A. Sakov, H. Shen, S. Zeltyn, L. Zhao. 2005. Statistical analysis of a telephone call center: a queueing-science perspective. Journal of the American Statistical Association 100 36-50.

Cleveland, B., J. Mayben. 2004. Call Center Management on Fast Forward: Succeeding in Today's Dynamic Inbound Environment. 15th Printing, Call Center Press.

Cottet, R., M. Smith. 2003. Bayesian modeling and forecasting of intraday electricity load. Journal of the American Statistical Association 98 839-849.

Diebold, F. X., C. Li. 2006. Forecasting the term structure of government bond yields. Journal of Econometrics $130337-364$.

Easton, F. F., J. Goodale. 2005. Schedule recovery: Unplanned absences in service operations. Decision Sciences 36 459-488.

Eckart, C., G. Young. 1936. The approximation of one matrix by another of lower rank. Psychometrika 1 211-218.

Efron, B., R. J. Tibshirani. 1994. An Introduction to the Bootstrap. Chapman \& Hall/CRC.

Gans, N., G. M. Koole, A. Mandelbaum. 2003. Telephone call centers: tutorial, review, and research prospects. Manufacturing and Service Operations Management 5 79-141.

Guerrero, V. M., J. A. Elizondo. 1997. Forecasting a cumulative variable based on its partially accumulated data. Management Science 43 879-889.

Harvey, A. C. 1990. Forecasting, Structural Time Series Models and the Kalman Filter. Cambridge University Press, New York.

Hoerl, A. E., R. W. Kennard. 1970a. Ridge regression: Biased estimation for nonorthogonal problems. Technometrics 12 55-67.

Hoerl, A. E., R. W. Kennard. 1970b. Ridge regression: Application to nonorthogonal problems. Technometrics 12 69-82.

Hur, D. 2002. A comparative evaluation of forecast monitoring systems in service organizations. 33rd Annual Meeting of the Decision Sciences Institute, San Diego, CA, USA .

Hur, D., V. A. Mabert, K. M. Bretthauer. 2004. Real-time work schedule adjustment decisions: An investigation and evaluation. Production and Operations Management 13 322-339.

Jolliffe, I. T. 2002. Principal Component Analysis. 2nd ed. Springer-Verlag: New York.

Jongbloed, G., G. M. Koole. 2001. Managing uncertainty in call centers using Poisson mixtures. Applied Stochastic Models in Business and Industry 17 307-318.

Kekre, S., T. E. Morton, T. L. Smunt. 1990. Forecasting using partially known demands. International Journal of Forecasting 6 115-125.

Mehrotra, V., O. Ozluk, R. Saltzman. 2006. Intelligent procedures for intra-day updating of call center agent schedules. Tech. rep., Department of Decision Sciences, San Francisco State University, (under review at Production and Operations Management).

Reinsel, G. C. 2003. Elements of Multivariate Time Series Analysis. 2nd ed. Springer.

Shen, H., J. Z. Huang. 2005. Analysis of call centre arrival data using singular value decomposition. Applied Stochastic Models in Business and Industry 21 251-263. 
Steckley, S. G., S. G. Henderson, V. Mehrotra. 2004. Service system planning in the presence of a random arrival rate. Tech. rep., Cornell University.

Taylor, J. W. 2007. A comparison of univariate time series methods for forecasting intraday arrivals at a call center. Management Science forthcoming.

Thompson, G. M. 1996. Controlling actions in daily workforce schedules. Cornell Hotel and Restaurant Administration Quarterly 37 82-96.

Wallace, R. B., W. Whitt. 2005. A staffing algorithm for call centers with skill-based routing. Manufacturing and Service Operations Management 7 276-294.

Weinberg, J., L. D. Brown, J. R. Stroud. 2007. Bayesian forecasting of an inhomogeneous Poisson process with applications to call center data. Journal of the American Statistical Association forthcoming. 